\title{
EVOLUTIONARY ASPECTS OF CIRCUMSTELLAR MATTER IN BINARY SYSTEMS
}

\author{
MIROSLAV PLAVEC \\ Dept. of Astronomy, Univ. of California, Los Angeles
}

\begin{abstract}
Several groups of close binary stars are considered in an attempt to explain their present state as a consequence of a large-scale mass transfer or mass loss in the past: Algol-like semidetached binaries, some shell stars (AX Mon), some binary X-ray sources (Cen X-3, Her X-1), the recurrent nova T CrB, helium-rich binaries $v$ Sgr and $\mathrm{KS}$ Per, and the symbiotic variables.

Algol-like binaries like U Sge cannot be products of a conservative case A of mass transfer; rather, mass loss from system and/or a temporary contact stage must be invoked. Nova T CrB as well as the symbiotic variables probably contain a mass-losing giant and a helium star, which again may be a product of a previous mass transfer of type B. Similarly, some of the X-ray sources may actually be binaries undergoing a second process of mass transfer. The systems $v$ Sgr and KS Per may contain helium stars expanding to the right of the helium main sequence, while the other component may be a rather inactive main-sequence star. Some shell stars may be products of mass transfer. Mass loss from convective envelopes is also discussed.

Loss of mass and of angular momentum from many binary systems must be anticipated. Behavior of the mass-accreting stars may often be decisive for the appearance and evolution of the system.
\end{abstract}

\section{Introduction}

A few years ago, I had a long discussion on close binary stars with Dr. Alan Batten. We could agree perfectly on only one conclusion: that the real hell for a sinful astronomer (if any such exists, of course) would be a round trip through the heavens. Knowing now how different were our ideas about Mars and Venus from what is revealed now by the space probes, can you imagine the mental torture of an astronomer who proposed models of $\beta$ Lyrae, UX Monocerotis, $\varepsilon$ Aurigae, HR 2142 etc., etc. and suddenly sees the reality? And yet, he tried so hard to interpret all the available data... The trouble is that we know so little about masses, radii, luminosities, even about the evolutionary stage in which a particular system is just now.

You will understand that I am trying to mollify your attitude towards the paper I am going to present. For my task can be summarized as follows: Find the exact ways by which a given binary system has arrived just at its present stage, of which we know next to nothing! In his remarks on Sahade's paper at the La Plata Symposium on Stellar Evolution in 1960 - which happened to be one of the last meetings he attended - Otto Struve said (Sahade, 1962):

With regard to the discussion of the evolutionary characteristics I take little less interest in it. To me, much of this is still vague and perhaps subject to a great deal of doubt, and while I have nothing to criticize, I have the feeling that we know at present very little about what is going on in many of these binary systems. It is awfully hard to say anything about how such binary systems will evolve. There were times in my own experience when I thought that perhaps the existence of gas streams would have a very significant effect upon the evolutionary track of a star. At other times I felt that those phenomena were superficial and that they did not seriously modify the evolutionary track. And I think there is no consistent answer. 
It is my intention to show that the propects of our understanding of evolution of binary stars may not be so gloomy today as they were twelve years ago. Before I attempt to show what has changed since Struve's times, let me answer briefly his dilemma whether gas streams are significant for stellar evolution or not. While we can no doubt find binary stars where circumstellar matter is only a superficial phenomenon, I think there is little doubt that in general circumstellar matter plays an essential role in the evolution of close binary stars. I have two simple arguments in favor of this point of view, both based on a statistical survey of eclipsing binaries.

First, it is interesting to realize that in order to be conspicuous, an eclipsing binary must violate the laws of single-star evolution. Photometrically conspicuous eclipsing binaries have the primary minimum deeper than, say, $1^{\mathrm{m}}$. This presupposes an occultation of a hot star by a cooler but larger companion. This cannot happen in a main-sequence pair. The Algol-like semi-detached binaries can display very deep minima, and in them a hotter main-sequence star is combined with a subgiant which is cooler, larger and of smaller mass - in clear violation of normal single-star evolution. Among the first 9 eclipsing binaries to be discovered, no less than 7 were of this type, according to a list compiled by A. H. Batten. Only one system of the nine is normal and detached, namely $U$ Ophiuchi. The remaining star is $\beta$ Lyrae. Although picked up as a bright variable, it is a foremost representative of eclipsing binaries which are spectroscopically conspicuous - due to the presence of circumstellar matter.

My second argument is based on two surveys of eclipsing binaries. Among 145 eclipsing binaries with somewhat reliable data and brighter than $8^{\mathrm{m}} .5$ at maximum light, I found (Plavec, 1968) 59 systems with both components on the main sequence. Most of them are simple detached pairs with little interaction. About $20 \%$ of these main-sequence binaries, however, are contact or nearly contact, i.e. with strong interaction and probably with circumstellar matter in significant quantities. Circumstellar matter is present or must have been present in the past in most of the remaining 86 systems, since they are: 52 semidetached binaries, 14 systems of the W UMa type, 2 with a Wolf-Rayet secondary, and 18 eclipsing binaries with a giant or supergiant primary. Only among these 18 systems a few can be detached and simple, with little interaction.

More recently, I have surveyed, with the collaboration of R. S. Polidan, all eclipsing binaries contained in Batten's catalog of spectroscopic binary orbits (Batten, 1967) with periods longer than $5 \mathrm{~d}$. There are 60 such systems. Twenty-two among them can be classified as Algols or related to them. Three are of the AR Lacertae type, i.e. a pair of subgiants not filling the critical Roche lobes but with rather prominent $\mathrm{H}$ and $\mathrm{K}$ emission indicating possible extended envelopes. Fourteen systems cannot be classified easily but components are certainly interacting ( $\beta$ Lyrae, UX Monocerotis, VV Cephei and W Serpentis are included in this group). Sixteen systems appear to be detached and simple; some are main sequence systems (such as RR Lyncis), a few are detached giant and supergiant systems which may have evolved as single stars ( $\zeta$ Aurigae is included here). Five systems are undecided. My estimate is that $70 \%$ of 
systems with periods longer than $5 \mathrm{~d}$ are either strongly interacting now or their evolution cannot be explained without large-scale mass transfer in the past.

Selection rules in both surveys eliminated eclipsing binaries with components below the main sequence, such as UX UMa or some U Gem stars and novae. These are clearly also unstable systems where circumstellar matter plays an important role. We can safely say that at least $60 \%$ and probably as much as $75 \%$ of all known eclipsing binaries are of the strongly interacting type, where circumstellar matter plays an important role.

Simple, well-behaving systems with little if any circumstellar matter can be found only on the main sequence and rather exceptionally among giants or even supergiants provided that such systems are sufficiently large to permit free expansion of the components. Pairs of very similar stars, quite frequent on the main sequence, are almost non-existent among the giant and supergiant systems.

In a recent article, Kopal (1971) used just this absence of similar pairs among giants as an allegedly strong argument "focusing attention on the weak points of the optimistic picture of the evolution of close binary systems based on the theory of largescale mass loss or transfer". In his colorful language, he writes:

Thus the old charm that equally massive stars are equal also in size - so manifest on the main sequence seems to lose its power soon after the star evolves away from the main sequence. It is almost as though some new factor - previously inactive - entered the control room of stellar evolution at this stage; but if so, we are as yet in the dark as to its identity (l.c., p. 529).

I think I should discuss this point here since Kopal repeatedly returns to it, saying that "This is an embarrassing question, and the answer to it still eludes us".

On the contrary, I think the explanation is quite simple. The evolution across the Hertzsprung gap and up the giant branch is very rapid, and is progressively much faster for stars of larger mass. Associated with this evolutionary phase is a large increase in radius. In Iben's models (Iben, 1967), the age of a star of mass $m$ at the top of main sequence can be approximated by the linear formula (for $2 \leq m \leq 6$ )

$$
\log t^{\mathrm{I}}=8.686-2.5(\log m-0.352),
$$

while for a red-giant tip we get similarly

$$
\log t^{\mathrm{II}}=8.771-2.66(\log m-0.352) \text {. }
$$

Take now for example AR Aurigae, a system which Kopal mentions as one of the good examples of a main-sequence system with nearly identical stars. According to Popper (1970) the masses of the components are

$$
\begin{aligned}
& \log m_{1}=0.41\left(m_{1}=2.57 m_{\odot}\right), \\
& \log m_{2}=0.36\left(m_{2}=2.29 m_{\odot}\right) .
\end{aligned}
$$

Inserting into (1) and (2), we get $t_{1}^{\mathrm{II}}=410 \times 10^{6} \mathrm{yrs}, t_{2}^{\mathrm{I}}=460 \times 10^{6} \mathrm{yrs}$. This means: 
the primary component develops into a helium-burning giant while the secondary star is still within the main sequence band; the primary reaches the red-giant tip 50 million yrs before the secondary ends its main-sequence evolution. Over a period of some 400 million yrs when both stars are relatively young, small difference of masses means small difference in radii. But once one of them crosses the Hertzsprung gap, its radius increases rapidly and considerably, and a small disparity in masses is sufficient to cause large disparity in radii, spectral types and so on. In fact, even a smaller difference in masses will have the same effect. Subtracting (2) from (1),

$$
\log t_{1}{ }^{\prime \prime}-\log t_{2}{ }^{1}=2.50 \log m_{2}-2.66 \log m_{1}+0.14,
$$

we find that a difference of 3 to $8 \%$ in masses is sufficient to bring the more massive star to the red-giant tip while its twin sister, almost identical but slightly lighter, is still a main-sequence star. The above figures hold good for stars between about $2 M_{\odot}$ and $5 M_{\odot}$, but are only insignificantly changed for other masses. Stars which are almost identical twins on the main-sequence become very different beyond it unless they are exactly identical. Naturally such systems are very rare, and among those enumerated by Kopal - $\beta$ Aur, WW Aur, AR Aur, Y Cyg, YY Gem - probably only YY Gem fulfills the above condition of virtual equality of the components.

While the absence of similar pairs among giants is in this way explained by the ordinary theory of single-star evolution, it is clear that a good deal of observed close binary systems cannot be explained without assuming large deviations from singlestar evolution. I think a very fruitful fundamental concept is the assumption that each component in a close binary system has only a finite volume available for its expansion, and once it reaches the limit of dynamical stability, it begins to lose mass. Theoretical treatment shows that in many cases this mass loss proceeds on a time scale much shorter than the nuclear time scale of stellar evolution, and that large quantities of mass are lost before the star manages to restore secular equilibrium. Here is the theoretical foundation of the theory of large-scale mass loss or mass transfer.

Actual calculations of these processes have been so far based on the assumption that the limit of dynamical stability is identical with the innermost contact surface of the Jacobian zero-velocity surfaces, or, as we briefly say, with the Roche limit. This may not be so if forces other than gravity are at play. How far this can affect the basic results of the theory of mass transfer is difficult to say. At times I am tempted to share some of Kopal's pessimism expressed in his article cited above. I will attempt today to apply current theory to various groups of binary stars. I hope this will stimulate further research, both theoretical and observational. If we eventually fail to explain the observed systems, we will have to change the theory.

\section{Survey of Theoretical Results}

The general picture of evolution of binary stars in the case of mass transfer will be given only very briefly here, since it was reviewed several times (Plavec, 1968, 1970a), 
most recently in a very brilliant exposé by Paczyński (1971a). The course and outcome of the process of mass transfer depend in the first place on the mass and evolutionary stage of the mass-losing component. Following Kippenhahn and Weigert (1967) it has become customary to distinguish cases - the term 'modes' would be more appropriate - A, B, and C. Case A occurs when the initially more massive component - I will call it consistently the principal component - reaches its limit of dynamical stability (henceforth simply called the Roche limit) while it still burns hydrogen in its core. When the principal component reaches the Roche limit later, at the stage when it is moving across the Hertzsprung gap and up the giant branch and burns hydrogen in a shell surrounding the hydrogen-depleted core, we speak of mode B. Mode $\mathrm{C}$ corresponds to cases where the Roche limit is reached when the star expands along the second giant branch, and as a rule possesses two energy-generating shells (hydrogen shell and helium shell). Which of these three modes will apply to a particular system depends on the masses of the components and their separation. A very convenient way of showing this dependence is to plot the period of the system against the mass of the principal component, as in Figure 1. Lines can be drawn to separate the modes, A, B, and C, respectively. The lines drawn in the Figure actually correspond to a mass ratio of 0.5 . For other reasonable values of the mass ratio they would not be significantly different. A system of any mass ratio $q$ can be plotted if instead of its actual period $P$ we plot a 'reduced' or 'normalized' period $P^{*}$ computed by the formula

$$
\log P^{*}-\log P=-0.144 \log 2 q .
$$

(C.f. Horn et al., 1969; or Plavec, 1968). The lines in Figure 1 were drawn using the recent models of stellar evolution by Paczyński (1970). Plotted in the diagram as full circles are some model binaries for which evolutionary sequences have been computed in detail.

Since a star's radius increases only slowly as it traverses the main-sequence band, case A occurs only within a very limited range of short periods, typically about $1 \mathrm{~d}$. In a rapid phase proceeding on a thermal time scale the principal component becomes typically a subgiant secondary of smaller mass. This subgiant evolves very slowly but eventually begins to rise up the giant branch, reaching the Roche limit for the second time. However, the separation of the components is always so small in case A that an expansion of the mass-accreting star may easily interrupt the evolution and lead to the formation of a contact system. This problem will be discussed in Chapter 3 .

Models located in the region labeled $\mathrm{Br}$ are stars with radiative envelopes and hydrogen-burning shells. Mass loss proceeds again on a thermal time scale. Besides the instability on the surface due to the presence of the Roche limit, the star has another instability at its center since the hydrogen-poor core becomes thermally unstable when it exceeds the Schönberg-Chandrasekhar limit. This latter instability eventually predominates and mass transfer does not stop until helium is ignited at the center and the expansion of the envelope is halted. The remnant of the principal star - essentially only the helium-rich core - then crosses the $\mathrm{H}-\mathrm{R}$ diagram and 


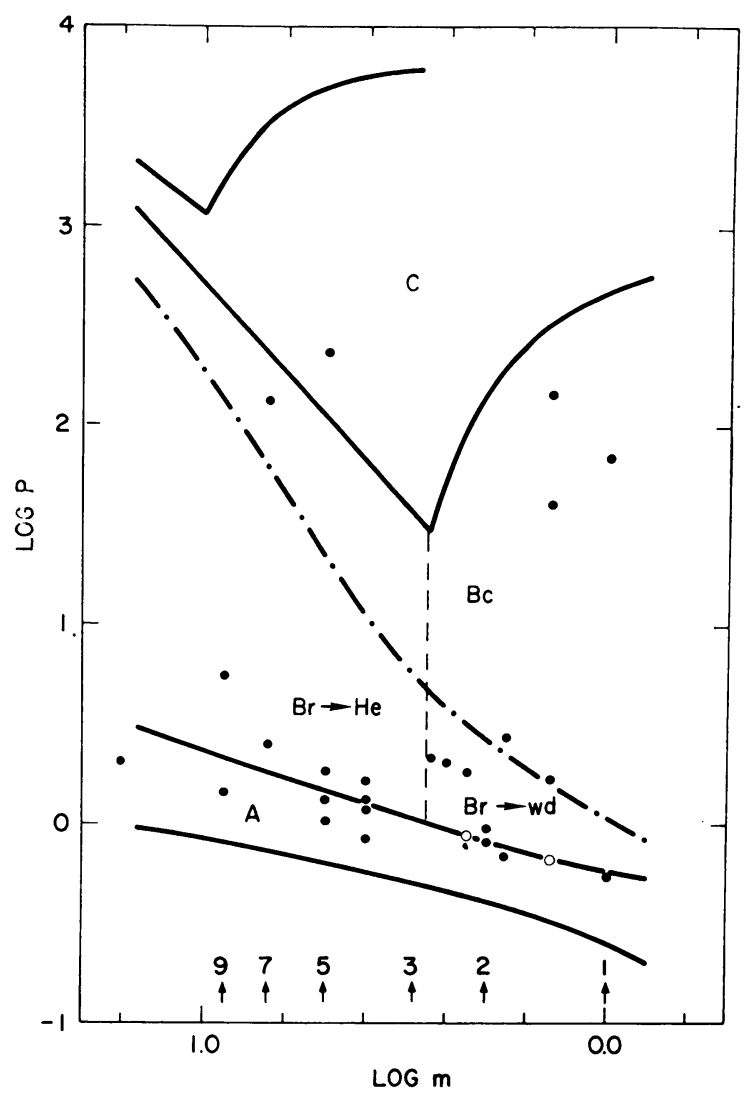

Fig. 1. The cases (modes) A, B, C, are shown here on a plot of the orbital period $P$ against the mass of the principal component $m$. Period is expressed in days, mass in solar units. Full circles indicate initial values for models that have been computed in detail. The two open circles indicate roughly the initial conditions at the beginning of the second phase of mass loss in case AB.

settles to a new equilibrium near the main sequence of the homogeneous helium stars.

Stars less massive than about $2.8 \mathrm{M}_{\odot}$, when trapped at the Roche limit soon after the end of the main sequence, have cores too small to ignite helium. The rapid phase of mass transfer gradually passes into a very slow phase where the contraction of the core and the associated expansion of the envelope are slowed down by increasing electron degeneracy in the core. Mass loss stops when practically no envelope above the hydrogen-burning shell is left. The remaining envelope virtually collapses on the star and the star becomes a white dwarf. In the stars of small mass $\left(<2.8 M_{\odot}\right)$ the mass of the core increases considerably as the star moves up the giant branch. Therefore, if such a star reaches the Roche limit at a later stage, it can still ignite helium and become a helium star rather than directly a white dwarf. Stars for which this can happen, i.e. those higher up on the giant branch, have by that time developed a deep convective envelope.

The region of deep convective envelopes is labeled $B c$. Very few models have been 
computed so far, and all of them very recently. These stars pose a specific problem since the mass loss from the principal component proceeds on a scale much shorter than the thermal time scale of the star. We shall discuss them in Chapter 5.

Finally, one case was computed for mode C (Lauterborn, 1970). The outcome was a massive white dwarf composed of carbon. The region $C$ will probably have more subdivisions with possibly different products of mass transfer. The upper limit of region $C$ is given by the ignition of carbon.

It would be interesting to plot actual systems into a diagram such as Figure 1. For most systems this is impossible since we do not know the masses. Transformed systems which already underwent the process are by no means eliminated from the graph. A second mass transfer is quite possible and I think that certain systems can best be explained in this way. I will refer to this possiblity when talking about the Prendergast-Burbidge model of X-ray sources in Chapter 6, about the helium-rich binaries $v$ Sagittarii and KS Persei in Chapter 7, and about the symbiotic variables in Chapter 8.

The variety of possible configurations is greatly increased if multiple processes of mass exchange are admitted. However, the observed systems display such a large variety that the theory of mass transfer must be generalized still in other respects. In the past several years, I have attempted to interpret some actually observed systems by means of theoretical models. The results are rather disappointing. Some binaries can be tentatively assigned to the theoretical types A, B or C of mass transfer. But the old Mephisto's saying proves again to be true: "Grau, teurer Freund, ist alle Theorie, und grün des Lebens goldner Baum". The real systems are enormously more complicated.

Interestingly, the star that is most frequently responsible for these complications is the mass-accreting component. No doubt the primary factor that brings complications into a binary system is the more massive component when it expands, becomes unstable and begins to lose mass. But it seems to play the role of an éminence grise, a grey shadow behind the scenes. The mass loss appears to proceed rather smoothly, although this may be an illusion due to the circumstance that the mass-losing star, typically a red giant, is seldom prominent spectroscopically or photometrically.

Nevertheless, really conspicuous phenomena - outbursts including nova outbursts, flares, X-ray emission, radio flares, emission lines, complex absorption profiles most of these phenomena seem to be associated with the mass-accreting star. This star was almost completely neglected in the theoretical studies reviewed at the beginning of this chapter. This is certainly no longer possible, and recent identifications of binary systems with $\mathrm{X}$-ray and radio sources make it quite plain.

Another recent development with immediate impact upon the problems of evolution of binary stars are the attempts to discover collapsed objects in binary systems. The presence of a black hole was suggested for a number of binaries - $\beta$ Lyrae, $\varepsilon$ Aurigae, BM Orionis, HDE 226868. In $\varepsilon$ Aurigae there is probably enough space for a star to expand freely into the supergiant stage and thus to pass through all stages of normal stellar evolution. From this point of view, a collapsar in $\varepsilon$ Aurigae 
would not be a surprise. All the other systems are much too small. If they indeed harbor a black hole, we must postulate a shortcut in stellar evolution in the sense that the progenitors of the black holes never passed through the stages of supergiants. The mass transfer of mode $\mathrm{B}$ or $\mathrm{AB}$, in which heavy stars transform into helium stars, may be such a shortcut. Or else we must postulate a drastic reduction in the size of these systems, associated apparently with large mass loss and loss of angular momentum from the system.

It is still possible that the puzzles of $\beta$ Lyrae and BM Orionis will be explained without the black hole hypothesis. However, the problem of loss of mass and angular momentum from binary systems will remain since it emerges also when we want to interpret the Algol systems, some X-ray sources, and other objects.

In his review article quoted above, Kopal puts forward another argument in favor of mass loss from the system (1.c., p.534): The total mass of some present-day Algol systems is so low that the initial principal component is unlikely to have had enough time to evolve to the point of instability within the lifetime of the Galaxy, unless of course it was originally much more massive than the present total mass of the system indicates. It would be possible to argue successfully about some of the masses Kopal uses, since they are based on absolute elements published in his catalog (Kopal and Shapley, 1956), which have in many cases been superseded by more recent investigations. Thus R Canis Majoris, for which he gives $0.6 M_{\odot}$ as the total mass of the system, was found to have a total mass over $2 M_{\odot}$ by Sato (1971) and to be much less anomalous than it was thought once. Another system mentioned by Kopal as having total mass less than $1 M_{\odot}$, T Leonis Minoris, should not be used as an argument at all. Its spectroscopic elements (Struve, 1946) are based on three normal points each formed from a very scattered group of velocities corresponding nearly to the same phase. Struve says that they "are suitable only for a preliminary determination of the semi-amplitude of the velocity curve", and Batten (1967) justly assigns the lowest grade to this orbit. No mass determination should be based on such elements. Nevertheless, some of the Algol systems may represent an argument for mass loss from the system. However, I think we cannot go as far as to suggest that "... it is the mass loss, rather than a mere mass exchange, which seems to hold a clue to the actual situation" (1.c., p. 543). Even with Algols of low mass, the problem still remains that the less massive component appears more advanced in evolution being a subgiant often considerably overluminous for its mass - while the primary is a bright mainsequence star. This paradox, I think, cannot be explained without admitting some mass transfer between the two stars. It seems, however, that the process often is not conservative, i.e. that the total mass of the system and total orbital angular momentum are not preserved.

Virtually all papers dealing with mass transfer assumed the conservative mode, although the authors knew quite well that this may not be the best assumption, as was mentioned in almost every paper and recently emphasized in the review by Paczyński (1971a). The conservative case may not even be significantly simpler to treat than a more generalized case: the formula giving the instantaneous Roche radius 
to be compared with the stellar radius would simply be different. I think the conservative case has been so attractive mainly because it is physically well defined. It appears difficult to formulate another physically consistent model without a cumbersome hydrodynamical treatment of the gas flow in the system. Paczyński and Ziólkowski (1967) formulated a nonconservative model by introducing two parameters, one representing the fraction of mass lost from the system, and the other representing the rate of loss of orbital angular momentum. Simplified model calculations showed an interesting variety of results, but the authors themselves admitted that the choice of the parameters had been rather arbitrary.

Weigert and Lauterborn in 1970 formulated a physically well-defined and consistent non-conservative model in which mass was streaming out from the system through the Lagrangian point $L_{2}$. Large loss of angular momentum greatly decreased the size of the system and its period. It is to be regretted that the model has not been published.

In spite of an annoying lack of observational data on mass loss, and lack of hydrodynamical models of gas streaming, it is quite clear now that the study of nonconservative models is urgently needed. I hope to show it in the next two chapters.

\section{The Semidetached Binaries of the Algol Type}

By the Algol systems I will mean semidetached binaries in which the less massive component is apparently more advanced in evolution, i.e. it is located above the main-sequence band, usually in the region of subgiants of spectral types $F$ through $\mathrm{K}$. The more massive star is still on the main sequence and is of earlier spectral type.

(Incidentally, something should be done with the nomenclature on close binaries. It is very awkward to use the term "semidetached binaries of the Algol type", or any variant of it, to describe a class of eclipsing binaries so well defined that single word should describe it. However, I cannot use simply the term 'Algols' right from the beginning, since many authors still use the term 'eclipsing binary of the Algol type' to mean an eclipsing variable with constant light between minima. It has been argued several times (Kopal, 1955; Plavec, 1964) that this definition is ambiguous and does not specify a physically meaningful and homogeneous class of objects.

Case A of mass transfer - beginning when the primary component still burns hydrogen in its convective core - has often been invoked as a lucid example how a typical Algol system comes into being. Figure 2 is an example. The primary component reaches its Roche limit at $b$. As the rate of mass transfer increases quickly, the star moves down and to the right in the H-R diagram. Thermal equilibrium is not restored until at $e$ when the rapid phase of mass transfer ends. By that time the original primary is already the less massive component but is situated well above the main sequence in the region of the subgiants. Subsequent evolution proceeds on a nuclear time scale, thus providing us with much better chances actually to observe the system. The subgiant continues to fill its critical Roche lobe, and loses mass at a low rate, of the 


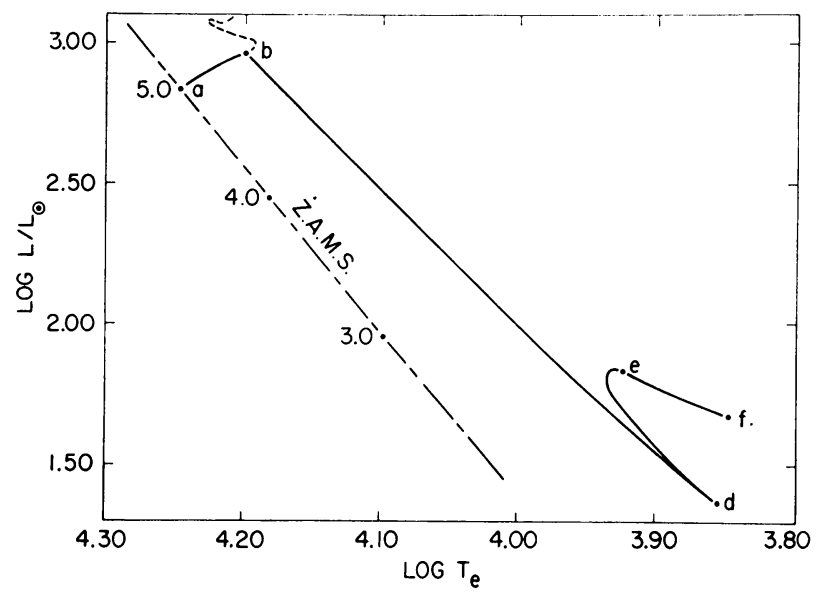

Fig. 2. Conservative mass transfer in case A. Mass outflow begins at $b$, both stars have equal mass at $d$, thermal equilibrium is restored at $e$, the subgiant begins to contract at $f$. Diagram drawn after Plavec et al., 1969.

order of $10^{-7} M_{\odot} \mathrm{y}^{-1}$ or so. The secondary star is now a bright massive early-type main-sequence star.

This picture represents the Algol systems extremely well if we are satisfied with a general qualitative picture. We have believed that most of the Algols are in the slow phase of mass transfer, between $e$ and $f$ in Figure 2, but that for example $U$ Cephei or RZ Scuti may be nearer to point $e$ or even between $d$ and $e$ because of more prominent gas-stream phenomena and rapid rotation of the early-type component.

One difficulty in explaining the Algol systems by mass exchange in case A was noted almost at the beginning, however. Many Algols have relatively small total mass of the system, less than $4 M_{\odot}$ - for example X Tri, RW Tau, TW Dra, RZ Cas and many others. Many of these systems are notable for large overluminosity of the subgiant - it lies well above the main sequence and is too luminous for its mass. Most striking are the cases of AS Eri, DN Ori and XZ Sgr, where the overluminosity amounts up to 5 to $10 \mathrm{mag}$. It is quite impossible to generate these luminous subgiants by the conservative case A process. Extensive calculations (Plavec and Horn, 1969; Plavec et al., 1969; Horn et al., 1969; Ziółkowski, 1970) can be summarized into the following 'rule of thumb': principal component tends to preserve the luminosity class in the process. A main-sequence B star has luminosity class IV when it has evolved some distance away from the initial main sequence; if it fills the critical Roche lobe at that time, it becomes a subgiant, luminosity class IV. Mass transfer starting very shortly before the B star terminates its main sequence evolution actually gives giants, and indeed the B star at such stage would be classified as luminosity class III. Among the A and F stars only luminosity classes V and IV or IV-V correspond to the main-sequence stage; and indeed, the transformed stars are luminosity 
class IV-V at best. The remnants of principal components of small mass - say less than $2.5 M_{\odot}$-invariably remain quite close to the zero-age main sequence.

This is why it has been suggested (Weigert, 1968) that Algol systems of small mass are produced by mass exchange of mode $\mathrm{B}$. In this case, the expanding primary reaches the Roche limit at the time when it burns hydrogen in a shell surrounding the helium-rich core where hydrogen has already been depleted. Thus at the beginning of the process the star is either traversing the Hertzsprung gap or climbing up the giant branch. These phases are much shorter than the main-sequence phase, but the star's radius increases so much that many more binaries should come under case B than under case A. However, our chances to observe such an object as a semidetached binary are good only if the mass of the system is sufficiently low, say below $4 M_{\odot}$. For more massive stars, the process is too short.

Compare the modes $\mathrm{A}$ and $\mathrm{B}$ for a star of $5 M_{\odot}$ in a binary system where the secondary component initially has $4 M_{\odot}$. If the initial period is $1.27 \mathrm{~d}$, the primary will reach its Roche limit when hydrogen at its center is depleted from $X_{c}=0.60$ to 0.15 but the convective core still exists and produces all the energy of the star. The rapid phase of mass transfer lasts 640000 years and the principal component transfers 1.82 solar masses to the secondary. In the ensuing slow phase the primary loses another $0.66 M_{\odot}$ but at a much slower rate since this phase lasts 20 million yrs. This is comparable with the main-sequence lifetime of the principal component, which was about 45 million yrs. Since the transformed system displays deeper eclipses, we believed that the phase of slow mass loss should be identified with observed Algol systems. (For a more detailed discussion of this case see Plavec et al., 1969.)

If the sytem is slightly wider to begin with, and the initial period is about $2 \mathrm{~d}$, the primary does not become unstable until after a hydrogen shell source is formed in its interior. This example of mode B was studied by Krríž (1969a). The rapid phase lasts $4.2 \times 10^{5} \mathrm{yrs}$, i.e. it is not much shorter than its counterpart in case $\mathrm{A}$, but the star loses much more mass, namely $4.06 M_{\odot}$. The slow phase that follows lasts only $6.6 \times 10^{5} \mathrm{yrs}$, and ends when the primary - now only $0.69 M_{\odot}$ - ignites helium in its core. The envelope contracts quickly and the star crosses the H-R diagram, changing from a red giant into a helium-burning subdwarf. The whole process lasts hardly longer than 1 million yrs as compared to the 21 million yrs in case A. Therefore it must be concluded that our chances are very low to catch such a system in the process of mass transfer.

Things are different, however, when the primary is initially less massive than about $2.8 M_{\odot}$. When the hydrogen-rich envelope is stripped off, the remnant is not massive enough to ignite helium. Its final destination is the region of white dwarfs. But before it contracts to this stage, the primary climbs up the giant branch for a long time. The rapid contraction of the core that so accelerates the evolution in the preceding case of a star of moderate mass is now perceptibly slowed down by increasing electron degeneracy in the core. This makes the slow phase favorably long. For example, for a primary initially of $2 M_{\odot}$ this slow phase when the system is semidetached lasts one quarter of the main-sequence lifetime (Kippenhahn et al., 1967) while for the 
above discussed star of $5 M_{\odot}$ the ratio was $1: 80$. Since the relative and absolute lifetimes are so favorable for systems of low mass, and since such binaries are more frequent than those of moderate mass (simply because stars of lower mass are more frequent), case B of mass transfer for systems of low mass appears a very adequate explanation of the existence of numerous Algol-type semi-detached binaries - except for one circumstance: The evolution is very slow towards the end of the process, when the primary is way up the giant branch. Therefore, among the observed Algols, those systems should be most frequent in which the late-type star is a real giant, very overluminous for its small mass. This does not appear to be the case. The abovementioned extreme overluminosities found in DN Ori, AS Eri and a few other stars are exception rather than rule, and typical overluminosities do not exceed $4^{\mathrm{m}}$ (Ziółkowski, 1969).

In order to remove this discrepancy, Ziółkowski $(1969,1970)$ suggested that many Algols are actually in the so-called mode AB of mass transfer. In discussing the case $\mathrm{A}$ for a star of $5 M_{\odot}$, I assumed that the slow phase ends when the subgiant detaches itself from the Roche limit. Actually, this introduces only a short break in the process of mass loss. The slow phase of case $\mathrm{A}$ is a continuation of the mainsequence evolution of the primary star. It has now much smaller mass, has lost part of its envelope, but hydrogen continues to burn in its core. When it is eventually exhausted, the star shrinks (as the more massive stars typically do) at the end of the main-sequence phase. However, very soon it begins to expand again when the hydrogen burning shell is established around the hydrogen-depleted core. Thus, in our special case, within about $3 \times 10^{4}$ yrs the primary expands to the Roche limit again and a new phase of mass transfer sets in. (Stars of smaller mass do not have the contraction phase at all.) Since the star now burns hydrogen in a shell, we have case $B$ of mass transfer. The process is soon dominated by the thermal instability at the star's center so that the outcome is the same as in the case B discussed above: when the mass-losing giant eventually ignites helium in its core, rapid contraction sets in and the star develops into a helium-burning subdwarf. The only significant difference between this case $\mathrm{AB}$ and the pure case $\mathrm{B}$ is the time scale. The process of mass transfer is much slower in case $\mathrm{AB}$. This is understandable, since the principal component enters phase B as a star of only $2.5 M_{\odot}$ so that the process must be considerably slower than for the $5 M_{\odot}$ in pure case B. Horn (1971) studied the case AB for a star of $5 M_{\odot}$. As usual in mode B, the process is very much the same independently of the initial mass ratio. Let us distinguish - somewhat schematically - between the subgiant phase (when the mass-losing star has luminosity class IV and is in the lower half of its giant evolutionary track) and the giant phase (when the star is on the upper part of its giant branch). The ratio of the corresponding lifetimes is $11: 1\left(18.5 \times 10^{6}\right.$ as against $1.7 \times 10^{6} \mathrm{yrs}$ ) in favor of the phase of lower luminosity. The primary component spends most of the time as a subgiant, only moderately overluminous for its mass. This is different from case B discussed by Kříž where the ratio of lifetimes is roughly $3: 1$ in favor of the stages of higher luminosity.

Ziółkowski $(1969,1970)$, who discovered case AB independently of Horn, realized 
that the difference in lifetimes might be significant for the probability of discovery. Since typical subgiants in Algol-type binaries show only moderate overluminosity, he suggested that many of them are actually undergoing mass transfer of type AB. His histogram of the distribution of observed semi-detached binaries as a function of the total mass seems to indicate the existence of two separate groups: massive binaries with a total mass larger than $5 M_{\odot}$, and low-mass systems with a total mass less than $4 M_{\odot}$. Since in this latter case the initial mass of the principal component is likely to be below $2.8 M_{\odot}$, the dichotomy appears rather natural. It was believed that the massive binaries are products of mode $\mathrm{A}$, while the low-mass group were binaries observed in mass transfer of type $\mathrm{B}$ or $\mathrm{AB}$; the ratio between these two groups remained rather uncertain.

The cause of the uncertainty was the problem how frequently can the case $\mathrm{AB}$ actually occur. In our survey of mass transfer of mode A from a star of $5 M_{\odot}$ (Plavec et al., 1969; Plavec and Horn, 1969), we found that the slow phase is terminated either by an overall contraction of the mass-losing component, or by expansion of the mass-accreting star which reaches its own Roche limit. Clearly only the former alternative permits the primary star to enter stage B of mass loss. This happens only if at the end of the slow phase the system is relatively large, and/or the secondary is not very much more massive than the primary. These favorable conditions obtain only when initially there was not a large disparity in masses $\left(m_{2} / m_{1}>0.6\right)$ and the primary star did not reach the Roche limit until shortly before the end of its main-sequence evolution (so that its central hydrogen content was already low to begin with, say $X_{c}<0.25$ ). Even if the primary component is allowed to embark on case B of mass loss, the process is so slow that most likely the other component becomes unstable before it can be terminated. Thus larger overluminosities can be attained only for a much narrower range of original conditions $\left(X_{c}<0.05\right)$. These stringent conditions lead us to suspect that case $\mathrm{AB}$ will be rather rare. Indeed, from private communications it seems that Ziółkowski also has largely retracted his original optimistic opinion on the cosmic importance of case AB.

Although the basic problem of the massive Algol-type systems (with total mass above $5 M_{\odot}$ ) appeared to be settled, I decided to take up the problem once more and from a different angle. Until now we have been satisfied with an overall, qualitative picture of the processes of mass transfer, and did not go beyond comparisons with statistical plots of certain quantities such as overluminosities of the subgiant components. I have now decided to compare theoretical evolutionary tracks directly with selected observed systems. This is not easy since observed characteristics of the Algol systems are as crude as our theoretical models. The semi-detached binaries are conspicuous photometrically and it is a serious problem to obtain reliable relative dimensions and inclination. Spectrographic observations are much more difficult since typically only one spectrum is observed and is often contaminated by gas streams. Batten spent a number of years trying to get better data for U Cephei. A similar effort was made in the case of U Sagittae - by Cester in Trieste photometrical- 
ly (Cester and Pucillo, 1972) and by Grygar and myself - at Victoria in 1969/70 spectroscopically. In neither case can the available results be considered final, yet some conclusions can be based on them with confidence.

I will show first that $U$ Sagittae cannot be explained by a conservative case $A$ of mass transfer. In the model by Cester and Pucillo, the total mass of the system is $5.7 M_{\odot}$ of which the primary - now a subgiant - has $1.6 M_{\odot}$ and the secondary has $4.1 M_{\odot}$. Assume now that the total mass of the system (and also the total orbital angular momentum) has been preserved. The initial mass of the primary must have been $3 M_{\odot} \leqslant m_{1}<5.5 M_{\odot}$. One plausible choice of initial parameters is $m_{1}=4 M_{\odot}, m_{2}=$ $1.7 M_{\odot}$. The configuration at the end of the rapid mass transfer can easily be predicted from the extensive tables by Horn et al. (1969). The outcome depends on three parameters, of which two - the initial masses - have already been fixed. Thus in Table I we can write down a family of solutions depending, for example, on the initial radius of the primary component (or its central hydrogen abundance):

TABLE I

Case A Models for U Sagittae

$\begin{array}{llllllll}X_{c 1} & \begin{array}{l}R_{1}{ }^{0} \\ \left(R_{\odot}\right)\end{array} & \begin{array}{l}m_{1}{ }^{e} \\ \left(M_{\odot}\right)\end{array} & \begin{array}{l}m_{2}{ }^{e} \\ \left(M_{\odot}\right)\end{array} & \begin{array}{l}P e \\ (\text { days })\end{array} & \text { Sp 1 } & M_{1} & \begin{array}{l}R_{1} \\ \left(R_{\odot}\right)\end{array} \\ \cdots & & & & & & & \\ 0.05 & 3.72 & 1.24 & 4.46 & 2.10 & \text { G1 IV } & +2 m 0 & 3.3 \\ 0.15 & 3.49 & 1.35 & 4.35 & 1.61 & \text { F6 IV } & +22^{m} 1 & 2.9 \\ 0.25 & 3.21 & 1.66 & 4.04 & 1.16 & \text { F0 IV } & +1^{m} \cdot 6 & 2.5 \\ \text { observed } & & 1.6 & 4.1 & 3.38 & \text { G3 IV } & +2^{m} \cdot 1 & 4.9\end{array}$

None of the three models is satisfactory. Small initial $X_{c}$ gives good spectral types but wrong masses; $X_{c}=0.25$ gives good masses but wrong spectral type. In all three models, the period is too short and the size of the subgiant too small. Subsequent slow mass transfer would affect the parameters in the correct direction, but it can easily be shown without much calculation that good agreement cannot be achieved. The conservative case presupposes that the orbital angular momentum

$$
J=G^{1 / 2} A^{1 / 2} \frac{m_{1} \times m_{2}}{\left(m_{1}+m_{2}\right)^{1 / 2}}
$$

is preserved. Combined with the constant total mass this yields the condition that

$$
C=A m_{1}^{2}\left(m_{t}-m_{1}\right)^{2},
$$

where $m_{t}$ is the total mass, is a constant throughout the whole mass transfer process, rapid or slow. Using present masses $m_{1}=4.1 M_{\odot}, m_{2}=1.6 M_{\odot}$, and present separation $A=16.9 R_{\odot}$, we find $C=728$. Inserting $m_{1}^{0}=4.0 M_{\odot}$ as a possible initial value, we get the initial orbital radius $A_{0}=15.7 R_{\odot}$. At the same time we know the initial mass ratio, therefore also the radius of the Roche lobe which the primary just filled. 
In other words, we get the initial radius of the primary, and this comes out to be $R_{1}^{0}=7.2 R_{\odot}$. This is clearly too much since at the top of the Main Sequence, the star of $4 M_{\odot}$ has a radius of only $3.73 R_{\odot}$ (for initial hydrogen abundance $X=0.60$; for $X=0.70$ we get $4.2 R_{\odot}$ ). Clearly the choice of the initial mass was wrong. But if we pick up any other initial mass within the permitted range $3 M_{\odot}$ to about $5.5 M_{\odot}$, we invariably find that the initial radius was too large for a main sequence star. A possible way out might be that the observed parameters of U Sagittae are wrong and do not represent any real system. I therefore tried a different set of parameters, based on Irwin's photometry and my rediscussion of Joy's spectroscopic observations of the subgiant and on McNamara's orbit for the bright component (cf. Plavec, 1967): total mass $7.8 M_{\odot}$, present masses $5.8 M_{\odot}$ and $1.9 M_{\odot}$, respectively, present orbital radius $18.8 R_{\odot}$. The differences with respect to Cester's newer elements show how poorly known are the characteristics of prominent Algol systems. This change introduced another dependence between initial radius and initial mass of the primary, as shown in Figure 3. However, the main conclusion is not changed at all: again, no main-sequence binary can generate the present system of $U$ Sagittae by conservative mass transfer in case A. Figure 3 shows that the same is trueof Z Vul, RS Vul, V 356



Fig. 3. Suppose the Algol system shown in this diagram originated by conservative case of mass transfer, mode A. Then the parental star must fulfill the indicated relation between its mass $m$ (in solar units) and radius $R$ (in solar units) at the beginning of mass transfer. We see that - except for $\mathrm{u}$ Her - the parental star could not be a main-sequence star, hence conservative mode $A$ is ruled out. 
Sgr and U Cep (where again the uncertainty is indicated by plotting two sets of elements, both contemplated by Batten - one based rather on spectroscopy, the other on photometry). Only u Herculis can probably be a product of case A, but this is not a very typical Algol system. Many systems of intermediate mass are not sufficiently well studied, but I have little doubt now: I think that conservative case A cannot explain the Algol systems. I shall discuss more direct physical arguments supporting this contention later in the text.

The problem is now: What shall we drop? The words 'case A' or the adjective 'conservative'? Let us consider the alternatives in turn. It is more difficult to review all possible intermediate stages in case B since no grid of models exist that would adequately describe the process. Fortunately, Harmanec (1970a) studied this case for a star of initially $4 M_{\odot}$ with companions of initially $3.2 M_{\odot}$ and $1.6 M_{\odot}$, respectively. The latter combination gives a total mass almost equal to that of Cester's model of U Sagittae. Let me quote values for two adjacent models from Harmanec's table (see Table II).

TABLE II

Case B Models for U Sagittae

$\begin{array}{lllllll}\begin{array}{l}\text { Age } \\ \left(10^{6} \mathrm{y}\right)\end{array} & \begin{array}{l}m_{1} \\ \left(M_{\odot}\right)\end{array} & \begin{array}{l}m_{2} \\ \left(M_{\odot}\right)\end{array} & \begin{array}{l}P \\ \text { (days) }\end{array} & \text { Sp 1 } & M_{1} & \begin{array}{l}R_{1} \\ \left(R_{\odot}\right)\end{array} \\ & & & & & & \\ 0.259 & 1.32 & 4.28 & 2.39 & \text { G1 IV } & +1 \mathrm{~m} 9 & 3.7 \\ 0.349 & 1.06 & 4.54 & 3.82 & \text { G2 IV } & +1 \mathrm{~m} 6 & 4.6 \\ \text { observed } & 1.6 & 4.1 & 3.38 & \text { G3 IV } & +2 \mathrm{~m} 1 & 4.9\end{array}$

A complete agreement would be very surprising in view of quite arbitrary initial conditions. I feel that in general this model in case B represents the observed system better than anything we could find in case A. Harmanec's model starts mass loss at the time when the primary component is not far from the Main Sequence: the initial radius, $4.78 R_{\odot}$, is only by $1 R_{\odot}$ larger than the maximum main-sequence radius. This is quite consistent with Figure 3 which shows that, if U Sagittae is an outcome of a conservative process of mass transfer, then the initial primary was probably $4 M_{\odot}$ or somewhat less and reached its critical radius not far from the main sequence. The same is apparently true of U Cephei, RS and Z Vulpeculae, and even for V 356 Sagittarii, although in this last case the parental star would be displaced somewhat farther away from the main sequence. The hypothesis that these Algol systems are products of mass transfer of the 'early B' type is favorably supported by the statistical survey of main-sequence detached systems I made in 1968 (Plavec, 1968). Among 45 eclipsing binaries of this type, 11 are so close that the principal component will eventually lose mass in type A process, while the majority, 34 , or $75 \%$, correspond to case B, many of them to 'early mode B'.

However, even without detailed model calculations, arguments can be presented against interpreting the Algol systems as products of a conservative case B. One is 
the predicted rate of mass loss. If $U$ Sagittae is approximately represented by Harmanec's models, the current rate of mass transfer would be about $3 \times 10^{-6} M_{\odot} \mathrm{y}^{-1}$, and the orbital period should secularly increase at a rate of nearly $1 \mathrm{~s} \mathrm{yr}^{-1}$. This is definitely refuted by the observations. Minima of $U$ Sagittae have been observed since the end of the past century, and analyzed for period changes by Svechnikov (1955) and by Cester and Pucillo (1972). The period seems to be shorter than it was most of the time in the past. We should therefore look for models which give secular increase of period about 2 orders of magnitude lower.

Another argument against accepting conservative case $B$ is the statistics of the Algol systems. The rapid phase of mass loss in case B is followed by a longer phase of slow mass transfer during which the mass-losing star is already a luminous giant, not a subgiant. For each binary like U Sagittae we should expect at least five binaries with luminous giants, bolometric magnitude between $+1^{M}$ and $-2^{M}$, spectral type G4 (for the fainter ones) to A7 (for the brighter giants). These systems would have periods between 10 and 25 days. Selection effects would be somewhat adverse to these binaries, since the bright main-sequence components have small fractional radii (0.12 to 0.05 ), hence eclipses would be less frequent, and also less deep, since the difference in surface brightness is smaller. The well-known dislike of the photometrists for longer periods (with the notable exception of Dr. Douglas Hall) would also disfavor such objects. There exist Algol systems with longer period, S Cancri, RS Cephei, RW Persei and others. Nevertheless I think we must conclude that we cannot find enough systems with bright giants to comply with the theoretical prediction.

A third argument against conservative case $B$ is the short duration of the process of mass transfer. The process is eventually dominated by the thermal instability of the helium-rich core, which develops almost independently of the conditions in the envelope. Therefore the mass loss takes about the same time as the normal evolution of the parental star up to the giant tip. Then why don't we observe any eclipsing binary systems in which the more massive component fills, or nearly fills, its critical Roche lobe? Our argument has always been that this phase is very much shorter than the phase of mass loss. This would not be true if the mass transfer were of type $B$.

We must conclude that the Algol systems like U Sagittae are products of nonconservative processes. Some material must be lost from the system, and carry away a part of the orbital angular momentum. But we need a definite physical picture how this happens in order to compute corresponding model sequence. Let us therefore consider a star of $5 M_{\odot}$ which fills its critical Roche lobe near the end of its main-sequence evolution. The star can be classified as about B5 IV, and has a radiative envelope How does it lose mass? Struve always spoke about prominences on its surface. I think solar physicists do not like this terminology. Solar prominences are not vehicles to carry away significant amount of material. The picture may be of some value for late-type components. We have no evidence about prominences on B stars. Kruszewski (1966) advocated the idea that the principal component rotates asynchronously and the material flies off along the tangent when it approaches $L_{1}$. I like more the idea that the Jendrzejec type of laminar flow applies here as soon as the star exceeds the 
Roche limit. There is probably no serious difference between the latter two alternatives. Material streams out at fairly low velocities, of the order of several $\mathrm{km} \mathrm{s}^{-1}$. Then it flows from $L_{1}$ towards the other component. In case $\mathrm{A}$, we always have a small orbital radius, so that the fractional radius of the other component is large. Benson (1970) studied the combination $5 M_{\odot}+2.5 M_{\odot}$, initial period $1 \mathrm{~d}$, which makes the orbital radius equal to $8.24 R_{\odot}$. Then the fractional radius of the mass-accreting component is 0.21 . So large a star will capture directly all in-coming material. Since the stream falls obliquely with a positive velocity component in the direction of rotation, the rotation velocity of the star will be accelerated. The impact area is heated. The crucial problem is how much of the kinetic energy of the stream is converted into heat and how fast the extra energy - in any form - is distributed through the star. Benson concluded that the radiative envelope is heated and assumes such a structure (deviating from radiative equilibrium) that as the accreted material is deposited, the star's radius increases rapidly. A transfer of only $0.1 M_{\odot}$ suffices in this case for the secondary to fill its critical Roche lobe, too, so that a contact system is formed. Dr. Ulrich at UCLA pointed out to me that Benson's solution is not necessarily the only one or the most likely one. It is conceivable that the falling stream invokes shear turbulence which transports a considerable amount of the acquired energy rapidly into the interior so that the radiative envelope is much less heated. Another factor probably acting in the same sense is the decrease in rate of mass loss from the principal component as soon as the secondary component does no longer increase its mass. I think that such a drop in mass outflow rate is bound to come. For it is not a rapid expansion of the principal star that drives the mass outflow in fact, the expansion is quite negligible - but rather the increasing radius excess over the Roche radius due to a rapid shrinking of the latter. But when the material is not deposited on the secondary component, the Roche limit will decrease much less rapidly. These 'delaying actions' may well take place but it seems that in most cases A eventually a contact system will be formed.

In this case, the future course of events may be quite different from the course chartered by computations describing the pure conservative case A. Very little is known about the structure and evolution of contact binaries, but recent activity has been very promising. From the brief reports published so far (Hazlehurst and MeyerHoffmeister, 1971; Biermann and Thomas, 1971) it is hardly possible to assess fully the impact of their work on the problem of the Algol systems - most attention is paid to the W UMa systems. From a paper by Moss (1971) and from a discussion with Dr. Whelan I gather that in contact systems the important problem is not only transfer of mass but also transfer of energy between the two stars. At a certain stage mass transfer may be reversed, go from the less massive star to the more massive one, and make the system detached again. Quite possibly this process must be studied if we want to understand the Algol systems.

On the other hand, we do not have any direct observational evidence in favor of one of our basic assumptions, namely that the mass outflow from the principal component is a quiet laminar outflow at low velocities, essentially sonic or subsonic. 
UX Monocerotis is a binary system not unlike the Algol systems, yet ejections of gas were observed from the late-type component with velocities of the order of $150 \mathrm{~km}$ $\mathrm{s}^{-1}$ to $250 \mathrm{~km} \mathrm{~s}^{-1}$ (Struve and Huang, 1958). If this is a rule rather than an exception, radical changes in our fundamental concepts will be necessary. Mass loss from the system will be an important factor.

One possible way of getting some insight into such a non-conservative process is to accept the formalism of Paczyński and Ziółkowski (1967) and introduce two parameters, $f$ and $g$, to describe the process. Suppose an amount of mater $\Delta m_{1}$ leaves the principal component. Then we assume that $f \Delta m_{1}$ escapes into space while $(1-f) \Delta m_{1}$ is intercepted by the secondary component. Clearly $0 \leqslant f \leqslant 1$, and $f=0$ is the conservative case. The escaping material carries away a certain amount of angular momentum $\Delta J$. Let us assume that

$$
\frac{\Delta J}{\Delta \Delta m_{1}}=g \frac{J_{0}}{M_{0}},
$$

where $M_{0}$ is the initial total mass of the system and $J_{0}$ the initial orbital angular momentum (at the beginning of mass transfer). If $g=1$, then $1 \mathrm{~g}$ of escaping material

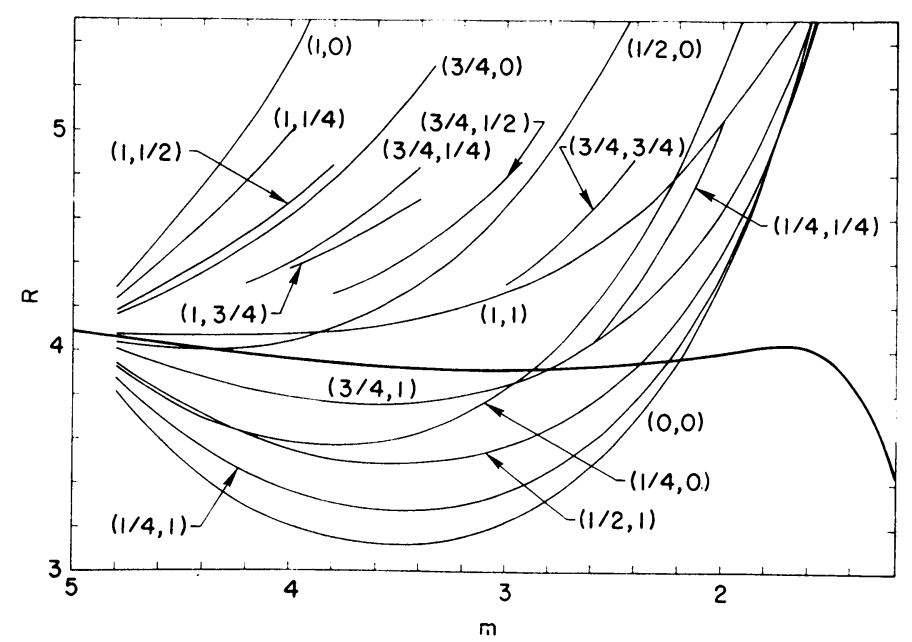

Fig. 4. The outcome of the rapid phase of mass loss of a non-conservative case A can be found always on the cross-section of the locus of thermal equilibrium models (heavy line) with the curve $(f, g)$ representing the variation of the Roche radius with mass.

carries away the initial average angular orbital momentum per $1 \mathrm{~g}$. The difficulty is with assigning a physically meaningful value to the parameter $g$. Clearly $g=0$ is unacceptable but $g>1$ is possible at least at early stages of the process. Most likely the parameter $g$ cannot be constant in real systems.

It is well known that configuration of the system at the end of the rapid phase of mass transfer of mode A can be obtained if we find the model for which the thermal 
equilibrium radius is equal to the Roche radius. This method was widely used by Paczyński and others, and many models similar to those we are going to use here were calculated by Plavec and Horn (1969), by Plavec et al. (1969), and by Horn et al. (1969). Figure 4 shows a diagram of the case when in a system initially consisting of a star of $5 M_{\odot}$ and a star of $3 M_{\odot}$, the principal component reaches the Roche limit when its central hydrogen content has dropped to $X_{c}=0.15$. The heavy curve is the locus of models in thermal equilibrium, with decreasing mass but fixed state of nuclear evolution in the interior. The arcs show the change of Roche radius of the principal component with its mass. They are labeled $(f, g)$ to indicate the dependence on the two parameters defined above. The instantaneous total orbital angular momentum $J$ is related to the instantaneous orbital radius and to the masses by

$$
A=\frac{J^{2}\left(m_{1}+m_{2}\right)}{G} \frac{m_{1}^{2} m_{2}^{2}}{m^{2}},
$$

but by the definition of $g$,

$$
J=J_{0}-\Delta J=J_{0}\left(1-g f \Delta m_{1} / M_{0}\right),
$$

and by the definition of $f$,

and

$$
M=m_{1}+m_{2}=M_{0}-f \Delta m_{1},
$$

$$
m_{1}=m_{1}^{0}-\Delta m_{1}, \quad m_{2}=m_{2}^{0}+(1-f) \Delta m_{1} .
$$

Subscripts and superscripts ' 0 ' refer to the initial parameters at the beginning of the mass loss. The instantaneous Roche radius of the mass-losing component is then

$$
R_{1}^{*}=A r_{1}^{*}\left(m_{1} / m_{2}\right)
$$

where the fractional Roche radius $r_{1}^{*}$ was calculated more accurately but can be approximated by

$$
r_{1}^{*}=0.38+0.2 \log \left(m_{1} / m_{2}\right) .
$$

Figure 4 shows how the intersections of the family of curves representing the Roche limit with the locus of the equilibrium models determines the end of the rapid phase. Plotted are curves for $g \leqslant 1$. Among them, the conservative case gives the largest mass transfer: the system is transformed from $5 M_{\odot}+3 M_{\odot}$ to $2.18 M_{\odot}+5.82 M_{\odot}$. Also, the rate of mass outflow will be largest since the excess of the equilibrium radius over Roche radius is a maximum for the conservative case.

However, modes with moderate mass loss from the system $\left(f=\frac{1}{4}\right.$ or $\left.\frac{1}{2}\right)$ and heavy loss of angular momentum $(g=1)$ come very close to the same final mass of the masslosing star. Indeed, for $g>1$, larger mass transfer and somewhat 1 tained. To represent the observed system of U Sagittae, we need $m$ 
$A=16.9, P=3.38 \mathrm{~d}, R_{1}=4.9 R_{\odot}$. The value of the radius shows immediately that none of our equilibrium models for $5 M_{\odot}$ can represent the present state of the system. Either the parental star had more than $5 M_{\odot}$, or the principal star has evolved rather far in the slow phase of mass transfer. Both alternatives may be true at the same time. We have not yet been fully successful in finding a model that would represent $U$ Sagittae satisfactorily. Also, even if we do succeed, the model will be acceptable only if the adopted values of $f$ and $g$ can be physically interpreted. However, the conclusion seems quite plausible now that U Sagittae, and probably many other Algol systems, are generated by processes of mode $A$ or quite possibly $A B$ in which mass loss from the system is not negligible, and in which phases of contact configurations may play an important role.

\section{Duplicity of Shell Stars}

Mass transfer in case B proceeds at a high rate, in particular in massive stars and in stars with convective envelopes. However, the outcome is rather modest. Take for example the case studied by Harmanec (1970a). The initial configuration is two B type stars, with masses $4 M_{\odot}$ and $3.2 M_{\odot}$, respectively, revolving in an orbital period of $1.8 \mathrm{~d}$. On the main sequence, such a system represents a well-observable spectroscopic double-line binary provided the orbital inclination is not too low. Eclipsing binaries U Ophiuchi and $\zeta$ Phoenicis are not much different from our model. Over a period of some $90 \times 10^{6}$ yrs it is transformed into a very unequal couple where the primary component (provided it accretes all the mass coming from its mate) is an early B-type star with $6.7 M_{\odot}$ while the remnant of the originally more massive star has only $0.5 M_{\odot}$ and a radius of only $0.2 R_{\odot}$. Spectroscopically and photometrically it would be difficult to detect that the B star is not single. The predicted rate of mass transfer reaches a peak value of nearly $10^{-5} M_{\odot} \mathrm{yr}^{-1}$ for our star of $4 M_{\odot}$, and can be much higher for more massive stars or for stars with convective envelopes. Moreover, spectroscopic binaries with B-type components and periods of the order of days or tens of days are rather commonplace. Spectroscopic phenomena associated with high-rate mass transfer or mass loss must be quite conspicuous. Relatively high frequency of systems that must be at this stage partly compensates for the short duration of the process. Therefore at least a few binary systems should be observed just at the stage when mass is streaming in the system. Where are they?

One would expect that the gas streams are most likely to be observed when they form a disk or an extended atmosphere around the mass-accreting component. The mass-losing star is a late-type giant with low surface brightness, rather difficult to observe if the system is not eclipsing. Also the gaseous streams between the two stars will in general not project on the disk of the bright component when the system is not eclipsing. The accumulation of material around the accreting star should be much easier to observe. It was this line of reasoning that led me in 1968 to suggest that some shell stars may actually be binary stars of this type. The peculiar eclipsing binaries $\beta$ Lyrae and V 367 Cygni have spectra which bear many resemblances to shell stars. 
A recent paper (Harmanec et al., 1972) seems to promote a hypothesis that all shell stars may be binaries. I think such a hypothesis is more difficult to defend. Physically the advantage of the binary model over a rapidly rotating equatorially unstable single star may be that the mechanism that supports the extended atmospheres is simply the continuous supply of material with excess angular momentum, coming from the other component. Observational evidence, however, lends little support. As a sample of well or fairly well observed shell stars let us take the list of 19 stars published by Underhill (1966). This list contains stars brighter than $7^{m}$. For the sake of completeness, let us add to it 4 Herculis, 88 Herculis, 14 Comae, and HR 2142, thus increasing the number to 23 . Among these 23 stars, 2 are certainly known to be binaries (17 Leporis, AX Monocerotis), and 6 others ( $o$ Andromedae, 88 Herculis, $\beta$ Monocerotis, $\phi$ Persei, $\zeta$ Tauri and HR 2142) are suspected of being binaries. Even if all of them eventually prove to be binaries, this would still be only $35 \%$ of the total number. It is estimated that at least half of all early-type stars are spectroscopic binaries. Thus the duplicity of these shell stars still might be quite accidental. Presence of a nearby companion may easily affect the shell phenomenon, but this still is not what we are looking for. The problem is whether at least in some shell stars the existence of the extended envelope is not only affected but directly caused by a companion of certain type.

Let us survey briefly the suspected systems. That of $\zeta$ Tauri displays radial-velocity changes that may be periodic with a period of $133 \mathrm{~d}$. However, according to Underhill (1952) the range is only $9 \mathrm{~km} \mathrm{~s}^{-1}$ so that the mass function is very small, about 0.01 . The star $\zeta$ Tauri is an early B-type star, and most likely its mass will not be very much different from $10 M_{\odot}$. The mass of the companion then might be $1 M_{\odot}$ to $2 M_{\odot}$ if the inclination is not too small. In a large system this companion will hardly play a significant role, and there is no evidence of its presence or influence. Delplace (1970) suggests that the radial-velocity variations are due to oscillations in the extended envelope. Underhill admits that the radial-velocity curve is based on the sharp shell lines rather than on the broad and shallow underlying photospheric lines, but points out that at the time when the star was studied the shell was stable and quiet. In any case, the shell is much more likely an intrinsic phenomenon.

Kř́ž (1969b) pointed out the agreement in the spectral type of the primary and in the orbital period between $\zeta$ Tauri and his model of mass transfer of mode B. In this case $\zeta$ Tauri would be interpreted as a system which recently finished mass transfer, so that one component is already a small helium star while the other has not yet accomodated all the material that the mass-losing component had supplied. There is no fresh supply now, however, and I suspect that this stage must be very short if it exists at all. So far no evidence has been furnished that would make this model more attractive than that of a single, rapidly rotating star.

Even less favorable for the binary hypothesis is the case of $\beta$ Monocerotis. Possible duplicity was recently suggested by Cowley and Gugula (1972). The period indicated by radial-velocity variations and certain other periodic or cyclic phenomena is $12.5 \mathrm{yrs}$. In this case the binary system is wide, i.e. no mass transfer can occur. Moreover, the 
mass function is very large, 12.5 , contrary to the case of $\zeta$ Tauri where it was very small. Cowley and Gugula find it very difficult to assign acceptable masses. Most likely the radial-velocity variations are not due to binary motion.

According to Hynek (1940) and Hickok (1971), $\phi$ Persei shows a repetitive radialvelocity curve which is, however, rather seriously and systematically distorted. Both investigators believe that lines of the secondary component can be seen at the helium lines $\lambda \lambda 4026$ and 4471 ; thus the secondary would also be a B star. Hickok's model, not very different from Hynek's, suggests two stars with masses $24 M_{\odot}$ and $12 M_{\odot}$, separated by $350 R_{\odot}$. Although the presence of such a companion will no doubt affect the distribution of mass in the system, the dimensions of the system are too large and the spectral types do not indicate that the extended atmosphere is formed by material coming from a thermally unstable component.

No radial-velocity curve exists yet for HR 2142. Suspicion of duplicity is based on surprisingly regular recurrence of a short-lived shell (Peters, 1972). The shell appears in cycles of about $81 \mathrm{~d}$, although with variable intensity and with somewhat variable duration, which is in each case very short, probably less than $10 \%$ of the period. Even if duplicity is demonstrated, the recurrent shell may be a consequence of modulation of the conditions in the extended atmosphere of the primary component by the companion, rather than to be due to gas streaming between the components.

The star $o$ Andromedae is a very controversial object. The shell appears sporadically with no conspicuous periodicity. Spectral lines are so broad that radial velocity variations found by different authors disagree. Schmidt (1959) concluded from his photoelectric observations that the star is actually an eclipsing binary with very distorted components very nearly in contact; the period indicated by his observations was $1.6 \mathrm{~d}$. Such a configuration could be a result of case A mass transfer leading to contact systems, and the envelope producing the shell spectrum would surround the whole system. Short-period radial-velocity variations were reported for EW Lacertae (HD 217050), for which also cyclic fluctuations of light were discovered by Walker (1953), and for 48 Librae (cf. a remark by Sahade, 1971). While unstable contact systems cannot be ruled out, atmospheric oscillations are a more likely explanation in all three systems including $o$ Andromedae for which eclipses have so far not been confirmed.

The star 88 Herculis was recently studied by Harmanec et al. (1972). They find that the radial velocities oscillate with a range of $10 \mathrm{~km} \mathrm{~s}^{-1}$ and a period of $87 \mathrm{~d}$. The mass function is small, $f(m)=0.008 M_{\odot}$. Since the spectral type is B6 IV-V, a mass of some $6 M_{\odot}$ is suggested for the visible component. Then the invisible star could have about $1 M_{\odot}$ and the separation would be $150 R_{\odot}$. A model of mass transfer of mode B studied by Harmanec (1970a) yields similar elements. The idea is that, as in the case of $\zeta$ Tauri, the secondary component is losing mass or was losing it relatively recently, and that this material now forms a ring around the blue component. So far there is no other observational evidence. I searched for the invisible star in the red and infrared spectral regions with the Lick 120-inch telescope, but the result was negative. Harmanec and his associates remark quite correctly that atmospheric 
oscillations may be an alternative explanation. The radial velocities were determined from the sharp shell lines, since the photospheric lines are too broad.

We come to the two shell stars in which duplicity has been firmly established. They are 17 Leporis and AX Monocerotis (HD 45910). These two systems are rather similar, since they combine a B star with a red giant. The question is whether this combination is accidental. I think that it is not, and that the instability of the red giant leads to gas streams which in turn produce the extended atmosphere around the B star - either directly, forming a ring, or indirectly, by interacting with the B star which then ejects matter as a secondary effect. Let us examine the evidence for this hypothesis. 17 Leporis was recently studied by Cowley (1967). The primary component is probably about B9, the secondary is about gM1 and its lines show up in the near infrared. Only the red star gives a satisfactory radial velocity curve. The relatively small value of the mass function, $f(m)=0.24$, indicates that the orbital inclination will probably be small. From a high-dispersion study of the $\mathrm{Mg}$ II line which is not directly affected by the shell but otherwise rather poor, Wright (1957) and Widing (1966) suggested that the mass ratio is about 4:1 or more, the blue component being the more massive. Cowley finds the following very tentative model as most satisfactory: $m(\mathrm{~B})=5.6 M_{\odot}, m(\mathrm{M})=1.4, i=24^{\circ}, R(\mathrm{~B})=2.6 R_{\odot}, R(\mathrm{M})=75$ $R_{\odot}, A=325 R_{\odot}$. In this model, the red giant becomes unstable at the time it approaches periastron. The mass outflow would then tend to have a pronounced periodical character since the eccentricity is fairly large, $e=0.13$.

Many shell lines occasionally develop double or multiple components, a phenomenon which was called 'outburst' by Struve. Major outbursts, according to Cowley, tend to occur between a quarter and a half of an orbital revolution past the time of periastron passage. The period is $260 \mathrm{~d}$, so the delay is between 65 and $130 \mathrm{~d}$. Our graduate student $\mathrm{R}$. Crawford calculated the travel time of particles ejected from the red star at periastron. This travel time depends very strongly on the ejection velocity. For velocities about $5 \mathrm{~km} \mathrm{~s}^{-1}$, the particles reach the vicinity of the blue star within about $45 \mathrm{~d}$. For velocities about $25 \mathrm{~km} \mathrm{~s}^{-1}$ the travel time is only about $25 \mathrm{~d}$. I think the result for the low velocities is encouraging and may indicate that the activity of the blue star is triggered by particles ejected from the red giant, perhaps as a consequence of convective overshoot. The greatest problem is how the streaming mass interacts with the blue star or with the material that already surrounds it. Specific to 17 Leporis are two difficulties: the masses are very poorly known, and the orbit is eccentric. For these reasons, AX Monocerotis appears to be easier to study, and our work on it will be reported in the next section.

\section{Convective Envelopes. AX Monocerotis and T CrB}

AX Monocerotis is included in lists of shell stars and Be stars, but its spectrum varies in a peculiar way, not typical for either group. The object is actually a binary system, in which an early $\mathrm{B}$ star and a $\mathrm{K}$ giant revolve in a period of $232.5 \mathrm{~d}$. The system was studied in detail by A. P. Cowley (1964), and also by Boyarchuk and Pronik (1967) 
and by Péton (1971). The early-type spectrum is that of a rapidly rotating B3: IV:star, but in particular the hydrogen lines are very complex and variable. As a rule they show emissions with P-Cygni profiles, but at times the absorption components are double or multiple. The early-type component is evidently surrounded by an extended variable atmosphere. Material is probably occasionally ejected by the star since for example $\mathrm{H} \alpha$ shows a nova-like profile, although the ejection velocities must be lower by a factor of three to four than in a real nova (Cowley).

At times, the star develops a shell spectrum with sharp absorption lines of ionized metals. Cowley found that this shell spectrum reappears periodically, although with variable intensity and duration. (Péton showed that the peak intensity of the shell lines varies rather smoothly with time and has been lower in recent years than in 1960). Shell spectrum is observed within the interval of phases 0.6 to $1^{P} .0$ or at most 1 . 1 , the phases being reckoned in units of the period with zero epoch at the time of conjunction with the $\mathrm{K}$ star in front (this would correspond to primary minimum if the binary were eclipsing). Boyarchuk and Pronik confirmed this periodicity and suggested that the extended atmosphere has the shape of a tail through which gas is streaming out of the Be star. However, the radial velocities of the shell lines do not lend much support to this interpretation since the difference with respect to the $\mathrm{Be}$ star, $10 \mathrm{~km} \mathrm{~s}^{-1}$, observed by Boyarchuk and Pronik is probably not significant and in any case cannot lead to the formation of the tail. I think the explanation advanced by Cowley is more promising, namely that material is streaming from the $K$ star into the vicinity of the $\mathrm{B}$ star. Negligible difference in radial velocities (actually observed only at a certain phase) may mean that the stream moves there approximately at right angles to the line of sight. This is in qualitative agreement with the general pattern of gas streams as indicated by the calculation of trajectories.

If we accept this hypothesis, then the $\mathrm{K}$ star plays an important role as a source of circumstellar material. Cowley suggested, much in accord with the ideas of Struve, that the $\mathrm{K}$ star is the seat of huge prominences. This may well be the case but I would like to point out that the present configuration in AX Monocerotis can hardly be explained without assuming a large-scale mass transfer in the past. The B star appears to lie near the main sequence, while the $\mathrm{K}$ star is a bright giant, perhaps $\mathrm{K} 2$ : II : . The radial-velocity curve of the $\mathrm{K}$ star is fairly reliably established. For the Be star, one must rely on the assumption that periodic displacements of the hydrogen emission lines represent the motion of the B star. While the mass ratio $m(B): m(K)=$ 2.5 is only a crude estimate, it would be difficult to disprove the conclusion that the $K$ star, although apparently more advanced in evolution, is the less massive component. In other words, AX Monocerotis can be considered as a large Algol system. It is tempting to complete this picture by assuming that the red giant actually fills the critical Roche lobe.

In the model I have considered (originally with Harmanec), the B star has $8.3 M_{\odot}$, the $\mathrm{K}$ star $3.2 M_{\odot}$, the orbital radius of the circular orbit is $360 R_{\odot}$ and the inclination is $66^{\circ}$. Adopting again a conservative case of mass transfer, we have a choice as to the initial mass of the principal component which is now the $K$ star. The range is 
about $6 M_{\odot}$ to $10 M_{\odot}$, and we adopted $7 M_{\odot}$. This arbitrary choice may naturally mean that our models will turn out to be rather far from the actually observed system, but one general feature of the problem will not be affected. Namely, with the long period of the system, it is inevitable that the principal component should begin to lose mass when it has a deep outer convective zone.

This brings about very serious difficulties in studying the process of mass transfer. In predominantly radiative atmospheres, the expansion of the outer layers due to reduced pressure absorbs so much energy that the radius decreases considerably. Therefore the condition can be fulfilled that the star always just fills the critical Roche lobe - in numerical model calculations we achieve this by proper choice of the rate of mass transfer. This is impossible for convective envelopes (Paczyński, 1965; Paczyński et al., 1969; Refsdal and Weigert, 1969; Paczyński and Sienkiewicz, 1972; Lauterborn and Weigert, 1972). Even if the mass of the star is decreased considerably, it is impossible to reduce the radius of the adiabatic envelope without somehow radiating away considerable part of its heat content. As soon as mass transfer to the other component starts, the Roche radius will decrease while the stars's radius will remain nearly unchanged. The star will overflow its critical Roche lobe. We believe that a laminar flow will build up under the pressure of the gas, and that gas will begin to stream through the nozzle at $L_{1}$ towards the other component. Jendrzejec (see Paczyński and Sienkiewicz, 1972) derived a formula where the rate of mass outflow depends, in the case of an adiabatic envelope, on the third power of the radius excess over the Roche limit. Since the Roche limit decreases rapidly while the star's radius remains nearly constant, soon after the beginning an extremely high rate of mass transfer is established. Paczyński and Sienkiewicz, using a rather simple but very ingenious method, found that in the case of a red giant originally of $1 M_{\odot}$ and $36 R_{\odot}$, the rate becomes as high as $0.13 M_{\odot} \mathrm{y}^{-1}$ !

Mass transfer in the model of AX Mon has been studied by R. S. Polidan and myself at UCLA. Knowing that such a high rate of mass transfer may not be supported by any observational evidence, we wanted to see if it can be cut down by introducing two additional modes of mass loss. Dr. R. K. Ulrich, at UCLA, derived a formula for convective overshoot based on his theory of convection in red giants. When a convective blob arrives into the vicinity of $L_{1}$, it may escape from the star because of its outward velocity. We believed that this process may be effective when the star just reaches the Roche limit but the radius excess is still too small for the Jendrzejec laminar flow to be important. In addition, mass loss was assumed to occur from the radiative atmosphere above the convective envelope. This thin atmosphere is nearly isothermal, and a modification of Jendrzejec's formula was applied here. A laminar outflow of material from the isothermal atmosphere is assumed to occur as soon as the atmospheric (surface) radius of the star exceeds the Roche radius, i.e. before the convective envelope begins to lose mass.

These two 'safety valves' proved quite inefficient. Figure 5 shows the evolution of the mass-losing star on the H-R diagram. The $7 M_{\odot}$ star begins to lose mass when its radius (from now on I will mean by this term the 'effective' or 'photospheric' radius) 




Fig. 5. Theoretical Hertzsprung-Russell diagram for the mass-losing component in the model of AX Monocerotis.



Fig. 6. Mass loss from the convective envelope of the principal component in the model of AX Monocerotis: Dashed line shows the variation of the critical Roche radius, while the full line shows the actual (photospheric) radius of the star. Calculations were made with a modified Paczyński's evolutionary code and cannot represent events occurring on a dynamical time scale.

is $108 R_{\odot}$, its luminosity $2228 L_{\odot}$, and $\log T_{e}=3.583$. By this time, the convective envelope begins $2.32 M_{\odot}$ outwards from the center, and reaches right up to the photosphere.

At the beginning, mass is being lost only from the radiative atmosphere. The rate is so low, not exceeding $10^{-7} M_{\odot} \mathrm{y}^{-1}$, that the star continues to move up along the giant branch, increasing its radius and luminosity for a while. In this way its radius soon exceeds the Roche limit and the rate of mass loss accelerates. This halts the 
normal undisturbed evolution, the radius ceases to increase but does not decrease either, see Figure 6. Since fairly large amount of material is now already being transferred to the secondary star (we assume the conservative case), the Roche limit shrinks, the radius excess increases, the process is self-accelerating. Within 1000 yrs, the rate of mass loss reaches a maximum of nearly 0.2 solar masses per year. Computations are very difficult at this stage and represent an extremely crude approximation only. Convergence was achieved only when the time step between two consecutive models was cut down to $5 \mathrm{yrs}$. The rate of mass loss remained higher than $10^{-2} M_{\odot} \mathrm{y}^{-1}$ for about $20 \mathrm{yrs}$. The maximum rate of mass loss indicates a time scale of the order of 15 to $20 \mathrm{yrs}$, which is very much shorter than the thermal time scale but still longer than the dynamical (pulsational) time scale. The thermal time scale is $6.3 \times 10^{3} \mathrm{yrs}$, the dynamical time scale is $2.2 \times 10^{-2} \mathrm{yrs}$, so that the time scale of the process at its peak happens to be just about the geometric mean between the two. These results are in full agreement with those obtained by Paczyński and Sienkiewicz (1972) for a giant of $1 M_{\odot}$ and $36 R_{\odot}$.

The rate of mass loss begins to drop when the principal component becomes the less massive star, since then the Roche radius of the mass-losing star increases again and the excess over the Roche limit diminishes. Some 1900 yrs after the beginning of mass loss, the Roche radius becomes again almost equal to the star's radius, and the surface instability is removed. However, the star remains thermally unstable since its core contracts and envelope expands, similarly as before the mass loss. The process is slower now since the mass of the star is only half the original mass. It continues to very nearly fill its critical Roche lobe, losing mass first at a rate of $7 \times 10^{-4} M_{\odot} \mathrm{y}^{-1}$, but slowing down. Mass loss from the convective envelope ceases when the mass is $2.6 M_{\odot}$, some 4000 yrs after the beginning of mass loss. Radius and luminosity increase steadily, but effective temperature decreases more than it would in undisturbed evolution. This phase lasts $10000 \mathrm{yrs}$, and at its end helium burning becomes so significant in the core that expansion is halted. The star reaches its maximum radius, $214 R_{\odot}$, and luminosity, $3130 L_{\odot}$, as an early M bright giant with mass $1.67 M_{\odot}$. It is brighter than a $7 M_{\odot}$ giant although it has only $24 \%$ of its mass. There are practically no losses of luminous flux by absorption since the star is in thermal equilibrium, and surface layers are already enriched in helium while the hydrogen abundance has dropped from $X=0.70$ to 0.58 . Mass loss from the radiative atmosphere continues for some time but at a negligibly low rate. Our computations have so far been carried up the point where the star's mass is $1.65 M_{\odot}$. The radius is already decreasing, the star moves to the left in the $\mathrm{H}-\mathrm{R}$ diagram, and we expect that it will move rapidly over to the region of helium stars.

In our model, the present configuration of AX Monocerotis, with the red star of $3.2 M_{\odot}$, corresponds to the relatively slow phase of mass transfer when the radius and luminosity increase again, and the rate is $4 \times 10^{-4} M_{\odot} \mathrm{y}^{-1}$. This material flows to the other star which is assumed to have a mass of $8.3 M_{\odot}$. If it is in thermal equilibrium, its radius is only 0.04 of the orbital radius. Therefore the gas stream does not fall on it but bends around and forms a ring. From the qualitative description of the 
gas ring model developed by Prendergast and Burbidge (1968) it transpires that part of the material of the ring will probably eventually be lost from the system while part is eventually accreted on the B star. Since the B star is so very much smaller than the $\mathrm{K}$ star, one must assume that the gas stream, although it tends to flow in the orbital plane, produces a ring or disk sufficiently thick perpendicular to the orbital plane to make the accrection roughly spherically symmetrical. Assuming that half of the material falls on the star, we must ask now whether a steady influx of some $2 \times 10^{-4}$ $M_{\odot} \mathrm{y}^{-1}$ can produce the phenomena observed in the spectrum of the $\mathrm{B}$ component. Much more critical is the problem whether the time interval of some $3000 \mathrm{yrs}$ has been sufficient for the B star to accomodate the $3.8 M_{\odot}$ of material transferred to it during the rapid phase. Loss of material from the disk means, of course, that the process cannot be strictly conservative, but this probably will not reduce the rate of mass outflow profoundly.

There is no doubt that the majority of close binary stars undergo this type of mass transfer since the increase in radius of the principal component is so large at the time it has a deep outer convective zone that it will most likely reach the Roche limit then. With the high rate of mass outflow the duration of the process is extremely short. However, the rate of mass transfer is so high that very conspicuous phenomena should occur and be occasionally glimpsed.

I suggest that the recurrent nova $T$ Coronae Borealis may be an example of the rapid process. Kraft (1958) showed that $\mathrm{T} \mathrm{CrB}$ is a binary consisting of a gM3 star and blue object which he classified as $Q$. The most remarkable thing is that the period of the system is 227.6 , nearly identical with AX Monocerotis. If the source of gas outflow is to be the $M$ star, then it must be a real giant, and possess a deep outer convective envelope. On the other hand, the blue object is probably not a degenerate star. Kraft found that the lower limits on the masses are 3.7 $M_{\odot}$ for the red giant and 2.6 $M_{\odot}$ for the blue object. This lower limit follows from the fact that the stars do not eclipse, so that the orbital inclination can hardly be larger than $70^{\circ}$. Paczyński (1965b) revised Kraft's masses downward and obtained as lower limits $2.6 M_{\odot}$ for the red giant and 1.9 $M_{\odot}$ for the blue star. But there is no compelling reason why the inclination should be as high as $70^{\circ}$. For $i=45^{\circ}$, for example, the total mass of the system and its size would be closely similar to our (and Cowley's) model of AX Monocerotis.

It is unlikely that the mass of the blue star could be as low as the Chandraskehar limit for white dwarfs. On the other hand, the red component is the more massive star in $\mathrm{T} \mathrm{CrB}$. If the system is at the stage of mass loss from the red giant, then it is near the beginning when the mass-losing star is still the more massive component. We must then expect a higher rate of mass outflow. Is the difference in behavior between the nova $\mathrm{T} \mathrm{CrB}$ and the peculiar Be star AX Mon due to this difference in mass influx? Or is it primarily caused by a different character of the mass-accreting stars? In AX Mon, the blue component may be more or less normal main-sequence star. In $\mathrm{T} \mathrm{CrB}$, Kraft estimates the color temperature of the blue star at $25000 \mathrm{~K}$ and derives a value of $0.2 R_{\odot}$ for the radius. In other words, the blue component would 
in this case be a helium star, and what we observe would be a second process of mass transfer. A more meaningful analogy would then exist between $\mathrm{T} \mathrm{CrB}$ and AG Peg. But again, the difference between a nova-like object such as AG Pegasi and the recurrent nova $\mathrm{T} \mathrm{CrB}$ might then again be in the rate of mass transfer.

Even if the proposed models for $\mathrm{T} \mathrm{CrB}$ and $\mathrm{AX}$ Mon are basically correct, I still have very grave doubts about the reality of the very high rates of mass transfer we get from theoretical calculations. I think rates smaller by some two orders of magnitude would still be sufficient for the models proposed here. But then - how to dispose of the theoretical predictions? One can easily argue that the model calculations are extremely crude. They represent an effort to carry the usual model-sequence calculations over a period when the star is unstable on a very short time scale - so short indeed that the computational codes used cannot reproduce the star's behavior properly. Interestingly different is the approach by Bath $(1969,1972)$ who found that the ionization zones in convective envelopes cause instabilities on the dynamical time scale. While his approach is quite different, his results are similar - again he calls for a sudden outflow of material at a high rate. Since the outburst is accompanied by a sudden increase of luminosity, he predicts U Geminorum-type outbursts of the cool component. Naturally, a subsequent violent reaction of the mass-accreting star is not precluded. The outburst is short-lived so that not much material is transferred in spite of the large rate; moreover, a good deal of the ejected material will probably be lost from the system. One such outburst does not solve the evolutionary dilemma of a giant trapped at the Roche limit. Our calculations reported above were made on the assumption that while our computing program cannot treat these dynamical instabilites, it may still give a good overall picture of the evolution of the system. Observationally, Bath's results also pose some embarrasing questions. Why don't we observe some of those outbursts in binaries of longer periods? Perhaps $\mathrm{T} \mathrm{CrB}$ is one example, and some symbiotic stars may be other similar cases. But in them, the blue component seems to erupt while the cool star probably does not change significantly. The problem of mass loss from convective envelopes is very puzzling.

\section{Case $B$ and the X-Ray Sources}

Mass transfer in case B invariably produces a star of small mass and radius - either a helium star or a white dwarf. In the conservative case, it leads to relatively large orbital radii and orbital periods. For example, the calculations by Kippenhahn et al. (1967) show that a system originally $2 M_{\odot}+1 M_{\odot}$ moving in an orbit with orbital period 1.15 is transformed into a main-sequence A star with $2.74 M_{\odot}$ and a low mass white dwarf of $0.26 M_{\odot}$, with an orbital period of $24 \mathrm{~d}$. Such a system will be very difficult to detect - spectroscopically or photometrically.

It is therefore very exciting to realize that a second mass transfer in such a system could produce an X-ray source. The problem was recently reviewed by Kraft (1972). The idea is that the kinetic energy of a gas stream falling on the mass-accreting component can be under suitable conditions converted into X-radiation of sufficient 
intensity. The maximum temperature that can be reached is

$$
T=1.5 \times 10^{7}{ }_{R}^{m},
$$

where both the mass $m$ and radius $R$ of the star are in solar units. This formula is very crude. The right-hand side should be multiplied by a factor easily as low as $10^{-2}$ since the efficiency of energy conversion is certainly very low. The UHURU satellite records radiation between 2 and $6 \mathrm{keV}$, so that the required temperature of such a hot spot is about $10^{7}$ to $10^{8} \mathrm{~K}$; in other words, the ratio $m / R$ should be roughly 10 to $10^{3}$ or so. This may still include neutron stars for which the ratio is about $10^{4}$. Also, mass transfer in case $\mathrm{A}$ or $\mathrm{B}$ is ruled out since the recipient is a main sequence star, but a second mass transfer in case B may be what we are looking for. The massaccreting star is then a white dwarf or a helium star. For the white dwarf obtained by Kippenhahn et al. (1967) the ratio $m / R=6$. The white-dwarf models by Hubbard and Wagner (1970) give the same value for a white dwarf of $0.20 M_{\odot}$, while for $1.05 M_{\odot}$ the ratio is about 13 . The helium star obtained by Harmanec (1970) gives $m / R=2.75$. A homogeneous helium star on the helium main sequence (Paczyński, $1971 \mathrm{~b}$ ) with a mass of $1 M_{\odot}$ has $m / R=5$ and this ratio increases with mass, reaching 10 for $8 M_{\odot}$. Massive helium stars seem to be almost equally promising as massive white dwarfs. Systems of low mass are naturally more common and Kraft points out that quite a significant fraction of observed X-ray sources are associated with the region of the galactic center, suggesting that they may be binaries of small mass.

Suitable temperature is of course not the only condition for a detectable X-ray source. The problem was studied in greatest detail by Prendergast and Burbidge (1968). In their model, the material is not accreted by the star directly. Rather, it first forms a ring around it, as it indeed must because of its excess of angular momentum. But viscosity tends to equalize the angular momentum across the ring. Therefore the outer particles will be accelerated and eventually some of them may excape from the system. The inner particles are slowed down and fall on the star. Prendergast and Burbidge were unable to investigate this final process. However, they find that that X-rays are generated in the inner ring where the gas is optically thin. The required rate of mass influx is $2 \times 10^{19} \mathrm{gm} \mathrm{s}^{-1}$, or $3 \times 10^{-7} M_{\odot} \mathrm{y}^{-1}$. If the rate were one order less, the mechanism would not work. Kraft explains that in U Geminorum stars and old novae the mass transfer is probably driven by emission of gravitational waves (Faulkner, 1971) rather than by thermal instability in the interior, since the masses are too small for significantly rapid nuclear processes. Hence the transfer rate is perhaps three orders of magnitude lower, and these systems cannot be expected to be $\mathrm{X}$-ray sources.

The rate of mass transfer is quite sufficient in case B when the mass-losing star is a giant of several solar masses. Indeed, I fear that the rate is in many cases somewhat too high. Paczyński (1971a) found that the maximum rate of mass transfer actually obtained in model calculations is very well reproduced if we simply divide the initial 
mass of the mass-losing star by its thermal time scale. This ratio can be written

$$
\frac{\mathrm{d} m}{\mathrm{~d} t}\left(M_{\odot} y^{-1}\right)=3.2 \times 10^{-8} \frac{R_{0} L_{0}}{m_{0}}
$$

where $m_{0}, L_{0}$ and $R_{0}$ are the initial mass, luminosity and radius of the mass-losing star. This simple formula gives good orientation values but cannot be exact since at the beginning of mass transfer its rate is affected by the rate at which the Roche limit shrinks, and this of course depends on the mass ratio and on the size of the system. Harmanec (1970a) found, for the same initial values of the principal component of $4 M_{\odot}$, a maximum rate of $9 \times 10^{-6} M_{\odot} \mathrm{y}^{-1}$ when the secondary has $3.2 M_{\odot}$, and $3 \times 10^{-5}$ when the secondary has $1.6 M_{\odot}$. The above formula gives $2.2 \times 10^{-5}$. Generally speaking, systems of low mass give values just about equal to the rate $3 \times 10^{-7} M_{\odot} \mathrm{y}^{-1}$ postulated by Prendergast and Burbidge, or somewhat lower. In the system $2 M_{\odot}+1 M_{\odot}$ studied by Kippenhahn, Kohl and Weigert, the maximum rate is $1.3 \times 10^{-6}$ but the average rate is much lower, $2 \times 10^{-7}$; Paczyński's formula gives $1 \times 10^{-6}$ for the maximum rate.

More massive systems will invariably give rates higher than $3 \times 10^{-7} M_{\odot} \mathrm{y}^{-1}$. The average rate in case of the system $9 M_{\odot}+3.1 M_{\odot}$ (Kippenhahn and Weigert, 1967) is $2 \times 10^{-4}$. Paczyński's formula gives $6 \times 10^{-5} M_{\odot} \mathrm{y}^{-1}$ for the maximum rate. This is two orders of magnitude more than postulated by Prendergast and Burbidge. They assumed that the mass-accreting star has half a solar mass and 0.2 solar radius. They postulated this star to produce $10^{35}$ ergs of $\mathrm{X}$-rays per second at a temperature of about $40 \times 10^{6} \mathrm{~K}$, and concluded that for this case the influx rate must be $2 \times 10^{19}$ $\mathrm{g} \mathrm{s}^{-1}$, which is $3 \times 10^{-7} M_{\odot} \mathrm{y}^{-1}$ (and not $3 \times 10^{-6}$ as they, and also Kraft, say). We may conclude that a helium star of 0.5 or $1 M_{\odot}$, combined with a star of several solar masses which expanded to the Roche limit and is losing mass by way of mode $B$, fully satisfies the conditions of the Prendergast-Burbidge model and should be an $\mathrm{X}$-ray source. If a crude extrapolation is permitted, then a similar but more massive system appears even more promising.

Sco X-1 and Cyg X-2 may be systems built on this model but the complexity of observed phenomena makes it very uncertain. Very interesting is the periodic source Cen X-3, which is an eclipsing binary according to Schreier et al. (1972). The X-ray source is eclipsed periodically in a period of 2.087. Short period (4.8 s) X-ray pulses are modulated periodically with the same period. If this modulation is interpreted as the Doppler effect, the system can be treated as a single-spectrum binary, and the mass function is $15.4 M_{\odot}$, very large indeed. Attempts to establish limiting elements of the system were made by van den Heuvel and Heise (1972), by Wilson (1972), and by McCluskey and Kondo (1972). Unless we make some assumption as to the nature of the system, the limits are too wide and they reveal very little. Our interest here is narrower; we ask whether Cen X-3 can be explained by the Prendergast-Burbidge model. The X-ray source must be a white dwarf or helium star, while the other component must fill its critical Roche lobe. In addition, we can assume 
that the radius of the $\mathrm{X}$-component is very small compared to the radius of the occulting star. Van den Heuvel and Heise found an upper mass limit $0.70 M_{\odot}$ for the pulsar, and $17 M_{\odot}$ for the unseen companion provided $i=90^{\circ}$. If $i$ is less than this, both masses are smaller. Wilson found that the upper limit on the mass of the pulsar can be put at $0.23 M_{\odot}$. Mass ratios and hence masses derived via the Roche limit depend very sensitively on the value of the Roche radius and I do not think that the arguments in favor of a very small mass are really compelling. There is little doubt however, that the mass of the unseen star is large. According to McCluskey and Kondo, if the X-ray source has less than $3 M_{\odot}$, the other star must have at least $15 M_{\odot}$. Such a system no doubt fulfills the conditions of the Prendergast-Burbidge model, but is rather acutely embarrassing to people who calculte evolution of close binaries. For a second process of mode B the period of 2.087 is uncomfortably short.

Van den Heuvel and Heise attempted to evade this difficulty by constructing a rather elaborate scheme. After the first mass transfer, a helium star of $4 M_{\odot}$ is formed, which subsequently explodes as a supernova and leaves a neutron star with about $0.5 M_{\odot}$. This star is now receiving mass from its mate which has about $15 M_{\odot}$. The regular $4.84 \mathrm{~s}$ pulses of the X-ray source are interpreted here as due to rotation of the neutron star with an embedded dipole magnetic field. As already pointed out by Kraft, there is a simpler way out, preserving the assumed white-dwarf or helium-star character of the X-component. We must assume that the first process of mass transfer was not conservative, that some mass escaped from the system and carried away part of the orbital angular momentum, hereby diminishing the orbital period.

Let me remark here that the optical identification of Cen X-3 with the eclipsing binary LR Centauri can now be practically definitely discarded. The periods, 2.087 and 2.095 respectively, are firmly established and incompatible. The error box defining the uncertainty in the position of Cen X-3 has now been considerably reduced and no longer includes LR Cen. The spectrum of LR Centauri was studied by M. S. Bessell (1972) who claims that the star that occults the X-component during the eclipses observed by UHURU is indeed peculiar and thus LR Cen should be seriously considered as the optical counterpart of Cen X-3, in spite of the discrepant position and period. However, Bessell's arguments are not convincing. He found a B8.5 III star with a rotational velocity $v \sin i=300 \mathrm{~km} \mathrm{~s}^{-1}$. He assumes that the star is rotating synchronously with orbital revolution, and obtains then large radius, large mass, high luminosity and concludes that the star is filling the critical Roche lobe. However, the assumption of synchronism is hardly warranted in this case. In Algol systems, the primary components tend to rotate faster than synchronism requires, at times very much so (Plavec, 1970b; van den Heuvel, 1970): U Cephei is a system with similar period and similar high velocity of rotation for the primary, which is, however, much smaller than the Roche limit and does not rotate in synchronism. I think that LR Cen may be a normal Algol-type system; there is nothing in the spectrum as described by Bessell that would indicate a really peculiar system.

Another periodic and probably eclipsing X-ray source is Her X-1. According to Tananbaum et al. (1972), in this case the period is 1.700 , and the mass function 
$f(m)=0.85 M_{\odot}$. Since the system is eclipsing, the inclination cannot be too low, so that the small value of the mass function must be primarily due to much smaller mass of the unseen component, compared to Cen X-3 .The variable star HZ Herculis appears to be the optical counterpart of Her X-1 (Liller, 1972; Lamb and Sorvari, 1972; J. and N. Bahcall, 1972). Photometric and spectroscopic evidence begins to build up that $\mathrm{HZ} \mathrm{Her} \mathrm{is} \mathrm{an} \mathrm{eclipsing} \mathrm{variable.} \mathrm{If} \mathrm{so,} \mathrm{the} \mathrm{Prendergast-Burbidge} \mathrm{model} \mathrm{may}$ well apply. The difficulty with too short a period is repeated here, although less strikingly because the masses are considerably smaller.

Finally, we have the case of Cyg X-1 which has been tentatively identified with HDE 226868, and with the radio source that 'turned on' in April 1971. HD 226868 is a spectroscopic binary with period 5.6. Its spectrum is that of a B0 Ib supergiant, rather surprising for a binary with such a relatively short period. The mass function has been found to be $f(m)=0.12$ by Webster and Murdin (1972) or 0.16 by Bolton (1972a). Most recently, Bolton (1972b) and Brucato and Kristian (1972) announced that the emission line of $\mathrm{He}$ II at $\lambda 4686$ shifts in antiphase to the absorption lines of the supergiant. If this line comes from the region surrounding the $\mathrm{X}$-ray component, then the mass ratio is about 1.4 or 1.5 . If the $\mathrm{B} 0 \mathrm{Ib}$ star is a normal supergiant with mass about $20 M_{\odot}$, then the secondary must be about $13 M_{\odot}$. While the X-ray source may be a region heated by falling material which streams from the supergiant, the secondary component cannot be a white dwarf or a neutron star. Bolton and others suggested that it has many properties of a black hole.

If so, then the star that collapsed into a black hole could not have passed through the normal supergiant stage of stellar evolution since the supergiant component observed today is too close, only some $43 R_{\odot}$ away. A star stripped first of its hydrogen envelope in mass transfer of type B can evolve without ever becoming a red supergiant. Again, this first mass transfer and the supernova outburst that may be anticipated during further evolution create some problems in accounting for the total mass of the system. Evolution with loss of mass and angular momentum is almost inevitable. However, observations are still to unreliable to guarantee the masses upon which all the speculation is built.

\section{The Helium - Rich Binaries}

Two stars with pronounced hydrogen defficiency in their atmospheres are singlespectrum spectroscopic binaries. They are $v$ Sagittarii and KS Persei (the latter is perhaps better known as HD 30353 or Bidelman's star). Since the stars show signs of circumstellar mass, it is tempting to explain their photospheric hydrogen deficiency by large-scale mass loss. Indeed, a process such as mass transfer in case B appears to be the easiest way to produce a helium-rich and hydrogen-poor star. However, case B cannot explain the observed degree of hydrogen depletion. While a considerable part of the hydrogen-rich envelope is stripped off, the process never reaches deep enough. Kippenhahn et al. (1967) and Kříz (1969a) showed that the mass transfer stops when the envelope has been stripped off down to the layers where the hydrogen abundance is about half the initial value. Such a mild hydrogen deficiency cannot 
be recognized spectroscopically. In $v$ Sagittarii, hydrogen abundance is down by a factor of about $10^{2}$ while KS Persei is even more extreme and the factor is about $10^{4}$ (Hack, 1967).

The simple case B is therefore ruled out. Much less is known about case C, but the one example studied by Lauterborn (1970) also does not lead to an extreme hydrogen deficiency. Towards the end of the mass transfer, a very tenuous envelope (in terms of mass) with mild hydrogen underabundance is left above the hydrogenburning shell source. But this shell is an effective barrier for convection, so that the really hydrogen-poor material from deeper layers cannot be mixed into the atmosphere unless the hydrogen-burning shell dies off. Lauterborn did not follow the process into these crucial phases. Cases B studied by Harmanec (1970a) and Horn (1971) seem to offer some clues. There the shell was still active when the envelope above it collapsed and the star was transformed into a helium star near the helium main sequence. Horn points out that the outermost envelope may become rotationally unstable after the collapse. Since no additional energy seems to be available, it is unlikely that the envelope could be dissipated in this way. One may surmise that the surface hydrogen depletion will become much more complete by convective mixing in the subsequent evolution when the helium star expands again and returns into the giant region of the $\mathrm{H}-\mathrm{R}$ diagram.

No model was followed completely through the stage of a helium star. Moreover, the helium stars obtained by Harmanec and Horn have small masses $\left(0.5 M_{\odot}\right)$ and cannot be expected to move into the giant region again. Paczyński (1971b) found that homogeneous helium stars move into the red giant region (provided the universal Fermi interactions exist) if their masses lie between about $0.9 M_{\odot}$ or $1.0 M_{\odot}$ on one side and $2 M_{\odot}$ on the other side. Biermann and Kippenhahn (1971a, 1971b) found a wider range of masses for such models. If the models have a degenerate core and a helium-burning shell, the range is $0.6<m / M_{\odot}<2.5$; if carbon burns in the core, the range is $1.0 M_{\odot}$ to $3.6 M_{\odot}$. Their models are simplified stationary models, while Paczyński's represent a true time-dependent evolutionary sequence. Neither set is entirely adequate for our purpose since it is assumed that even the outermost layers are completely devoid of hydrogen. However, it is very tempting to see whether $v$ Sagittarii and KS Persei could be roughly interpreted as evolved helium stars.

We do not have enough reliable data on these two stars, but a crude model will be attempted here.

The radial-velocity curve of $v$ Sagittarii is apparently reliable (Seydel, 1929), although a modern revision is desirable. The mass function is $f(m)=1.58$. Orbital inclination is much more uncertain. The star was reported by Gaposchkin (1945) to display partial eclipses. The eclipses are very shallow; the depths found by Gaposchkin are 0.15 and 0.08 respectively, while the probable error of his photographic estimates was \pm 0 . 20 . Obviously no elements can be based on such observations. In particular, no weight should be given to Gaposchkin's estimated inclination $i=47^{\circ}$. This was based on the assumption that both components are of equal size. The period of $v$ Sagittarii is $138 \mathrm{~d}$, and systems of such long periods tend to have components of 
very unequal radii, which is quite understandable if we recall our discussion of differential stellar evolution. Limited photoelectric observations reported by Eggen et al. (1951) confirmed the existence of eclipses and the fact that the invisible component is eclipsed at primary minimum. The invisible star is therefore hotter, and it is reasonable to assume that it is much smaller than the visible star. The mere existence of eclipses then indicates that the orbital inclination cannot be very far from $90^{\circ}$, and $\mathrm{I}$ think $i=80^{\circ}$ is quite a plausible assumption.

Keeping in mind that our working hypothesis postulates the mass of the helium star to be about $1 M_{\odot}$ to $1.5 M_{\odot}$, we get the set of acceptable masses shown in Table III.

TABLE III

Possible Masses of $v$ Sagittarii

\begin{tabular}{llll}
$m_{\text {invis }} / m_{\text {vis }}$ & $\begin{array}{l}m_{\text {vis }} \\
\left(M_{\odot}\right)\end{array}$ & $\begin{array}{l}m_{\text {invis }} \\
\left(M_{\odot}\right)\end{array}$ & $\begin{array}{l}A \\
\left(R_{\odot}\right)\end{array}$ \\
\hline & & & \\
2.0 & 1.98 & 3.95 & 62.0 \\
2.5 & 1.31 & 3.29 & 67.5 \\
3.0 & 0.99 & 2.98 & 70.9 \\
4.0 & 0.66 & 2.62 & 75.6
\end{tabular}

From Table III it follows that the invisible component must be the more massive star. This dilemma is well known in cases such as $\beta$ Lyrae or $\varepsilon$ Aurigae and led some astronomers to the suggestion that the invisible star is a collapsed object, a black hole. I do not think that any such hypothesis is needed here. At effective temperatures near $10000 \mathrm{~K}$, Paczyński's models have bolometric magnitudes near $-5^{M}$ or $-6^{M}$. If we assume that the other component is a main sequence star of about $3 M_{\odot}$, then it should be some five magnitudes fainter and yet its surface brightness can still be somewhat higher than that of the helium star.

I assume that the helium star is a remnant of an originally much more massive star which lost its hydrogen-rich envelope by the process of mode B. This type of process is suggested by the present period of $v$ Sagittarii. A star initially of $7 M_{\odot}$ gives in this case a helium-rich remnant of $1.13 M_{\odot}$ (Harmanec, 1970b). The process is not hampered by the $3 M_{\odot}$ companion which evolves much more slowly: it takes 250 million yrs to cross the main sequence band, while the $7 M_{\odot}$ star can develop into a giant, lose all its envelope, shrink into a helium main sequence star and expand again into a helium rich star, all together within not more than some 50 million yrs.

This picture, however, implies that almost all the material of nearly $6 M_{\odot}$ shed by the $7 M_{\odot}$ giant was dissipated into space rather than transferred onto the companion - for that star has today only $3 M_{\odot}$ and certainly started its life with not much less than that.

If my hypothesis is correct, we are witnessing a second mass loss from the same star, and this time it is the less massive component-a rather unusual event. An emission feature at $\mathrm{H} \alpha$, accompanied by a violet-displaced absorption, and other pecu- 
liarities in the spectrum indicate that mass loss is going on. Therefore the star should not be far from the Roche limit. The adopted mass ratio 0.37 and size of the system, $69 R_{\odot}$, put the Roche limit around the helium star at $20 R_{\odot}$. Its actual radius is unknown within rather wide limits. One source of great uncertainty is the effective temperature. The spectral type is usually given as $\mathrm{A} 0 \mathrm{p}$, but cannot well be used to derive effective temperature since the star's atmosphere has abnormally low opacity which enhances the metallic lines. This difficult problem was studied for the other helium-rich star, KS Persei, by Danziger, Wallerstein, and Böhm-Vitense (1967). They found $T_{\text {eff }}=10000 \mathrm{~K}$ and $M_{v}=-3^{M} .2$. KS Persei is usually classified as A5p, and is probably somewhat cooler than $v$ Sagittarii. If we take $T_{\text {eff }}=10000 \mathrm{~K}$ and $M=-5^{M} .3$ for $v$ Sagittarii, its radius will be $33 R_{\odot}$, much larger than the Roche limit. However, $T_{\text {eff }}=13000 \mathrm{~K}$, a more likely value, gives a radius of $17 R_{\odot}$. The model is crude but does not involve an open contradiction.

I think that complex observations of $v$ Sagittarii can verify and develop the model. Photometric observations can determine the difference in effective temperatures, even perhaps yield a crude estimate of the ratio of radii. Revision of the estimates of absolute magnitude and effective temperature is desirable and feasible with modern techniques.

Thanks to the effort by Wallerstein and his associates, KS Persei is actually better known although the star is fainter and circumstances less favorable. The system does not eclipse, so that orbital inclination is uncertain within wide limits. However, with very unequal components moving in a large orbit (the period is $360 \mathrm{~d}$ ), the inclination can still be fairly near $90^{\circ}$. I adopted tentatively $70^{\circ}$. For inclinations much smaller than this the masses are too large for almost any mass ratio. Table IV

TABLE IV

Possible Masses of KS Persei

\begin{tabular}{lllr}
$m_{\text {invis }} / m_{\text {vis }}$ & $\begin{array}{l}m_{\text {vis }} \\
\left(M_{\odot}\right)\end{array}$ & $\begin{array}{l}m_{\text {invis }} \\
\left(M_{\odot}\right)\end{array}$ & \multicolumn{1}{c}{$\begin{array}{l}A \\
\left(R_{\odot}\right)\end{array}$} \\
& & & \\
3.0 & 2.61 & 7.84 & 97.4 \\
4.0 & 1.72 & 6.89 & 103.9 \\
5.0 & 1.27 & 6.35 & 108.2 \\
6.0 & 1.00 & 6.00 & 111.3 \\
7.0 & 0.82 & 5.76 & 113.6
\end{tabular}

shows that for acceptable masses of the helium star, the mass ratio must be $5: 1$ or $6: 1$ in favor of the unseen companion. With a mass about $6 M_{\odot}$ a main-sequence star will have visual absolute magnitude near $-1^{M}$, while the visual absolute magnitude of the helium star was estimated at $-3^{M} \cdot 2 \pm 0.7$. Our explanation why the spectrum of the companion is not visible may still work. The bolometric absolute magnitude of the helium star is about $-3^{M} .5$, or about $1^{M} .5$ too faint for Paczyński's models. Combined with $T_{\text {eff }}=10000 \mathrm{~K}$ this gives a radius of $14.5 R_{\odot}$. In this model, the orbital radius is $111 R_{\odot}$, and with the mass ratio $\frac{1}{6}$ the radius of the critical Roche 
lobe of the helium star is $25 R_{\odot}$. Higher luminosity of the helium star would give better agreement with theoretical models as well as a star more nearly filling the Roche critical lobe. Again, the helium star can be considered as a remnant of a star originally of $7 M_{\odot}$. Since the companion is about $6 M_{\odot}$, the process might have been more nearly conservative, and a good deal of the mass lost by the principal component was probably captured by its companion. The main-sequence lifetime of a star of $6 M_{\odot}$ is, however, only about $35 \times 10^{6} \mathrm{yrs}$, while we estimated the age of the helium star at $50 \times 10^{6} \mathrm{yrs}$.

In spite of this discrepancy - which can probably be removed by adjusting the model slightly - the hypothesis that KS Persei is a helium star undergoing second phase of mass loss remains very attractive, in particular through the work of Wallerstein, Greene and Tomley (1967). They in fact proposed a similar hypothesis already five years ago. Studying the abundances of light elements in KS Persei, they found the ratio $\mathrm{H} / \mathrm{He}=10^{-4}$ in good agreement with the previous work of Nariai (1963). High abundance of nitrogen is suggestive of carbon cycling, but the two ratios found, $\mathrm{C} / \mathrm{N}=10^{-3}$ and $\mathrm{O} / \mathrm{N}=10^{3}$, are not easily reconciled. Wallerstein et al. prefer an evolution in which the central temperature originally was $24 \times 10^{6} \mathrm{~K}$ but subsequently dropped to $10-15 \times 10^{6} \mathrm{~K}$, with both stages lasting relatively long. Such a process is indeed conceivable, since this is exactly what happens in case A. The masses would be too low should we take the term 'central temperature' literally. Since Wallerstein et al. favor an initial mass about $5 M_{\odot}$, we should perhaps interpret the values given above as average temperatures in the convective core. A star of $5 M_{\odot}$ can spend some 30 to 40 million yrs on the main sequence, then reach the Roche limit and transform rapidly into a star of $2 M_{\odot}$ or even less. A long phase can follow in which the evolution is slow again. Since we postulate an ultimate loss of the whole envelope, the process must eventually pass over into the B mode; in short, we have a case $\mathrm{AB}$.

A great trouble of this picture is the long period, $360 \mathrm{~d}$. A very short initial period is required for case A to occur. Assuming for a while the conservative case, we can for example conjecture that the present configuration $1 M_{\odot}+6 M_{\odot}$ was originally $5 M_{\odot}+2 M_{\odot}$ or even $4 M_{\odot}+3 M_{\odot}$. But in this case the period could have increased by a factor of 8 while we need a factor of 360 . Even if we admit mass loss from system, it is difficult to find a physical model that would remove this discrepancy.

The two systems with helium-rich stars, $v$ Sagittarii and KS Persei, seem to be a rarity. This is not surprising since helium stars can develop into giants only if their masses lie within a very narrow range, perhaps narrower than $1 M_{\odot}$. On the hypothesis put forward here this also means a fairly narrow range in the masses of the parental stars.

The two helium-rich stars need not fill the critical Roche lobe at the present time and yet a non-negligible mass outflow from them is possible. As explained in the review paper by Dr. Böhm, helium stars have such a strong output of acoustical energy that very dense coronae are likely to be generated, and the mass outflow may come from these coronae. This idea has already been proposed by Nariai (1967). 
One last remark: $\beta$ Lyrae was reported to be hydrogen-poor, too (Boyarchuk, 1959; Hack and Job, 1965). According to Hack, hydrogen abundance is down by a factor of 10 . In this case the B8 II component of $\beta$ Lyrae would be rather similar to $v$ Sagittarii, and one could speculate whether or not the systems are in fact similar. But before we do so, the abundance determination for $\beta$ Lyrae should be re-examined.

\section{Symbiotic Variables}

Helium stars smaller than about $1 M_{\odot}$ or larger than about $2 M_{\odot}$ always remain to the left of the hydrogen main sequence. They have small radii and can hardly be expected to fill ever again their critical Roche lobes. In such systems, instability leading to mass transfer or mass loss can occur rather as a consequence of the secular expansion of the other component. Provided the helium star is a remnant of the initial principal component, we have a second process of mass transfer in the same system. This time, however, the one star is losing mass that originally accreted it. This may be considered as a kind of cosmic justice, only it is questionable whether the star that was originally robbed is capable of accepting the material back. From the available evidence one would conclude that the helium star does not accept the on-flowing material quietly, rather the interaction leads to a series of flares or minor outbursts which may occasionally attain a nova-like character. This, I think, is the basic explanation of the symbiotic variables.

A survey paper on the symbiotic variables was recently published by Boyarchuk (1969) who lists 21 objects which satisfy the following criteria: present in the spectrum must be absorption lines of a late-type component, as well as emission lines of $\mathrm{He}$ II, O III or similar ions; these emission lines should not be wider than about $100 \mathrm{~km} \mathrm{~s}^{-1}$; the light of the object can vary with an amplitude up to 3 mag. and in cycles with several years duration.

The symbiotic stars are rather faint objects, and while photometric data are incomplete, spectroscopic observations are scarce.

Nevertheless, we probably can sketch a general picture of behavior of the symbiotic variables. The late-type component changes very little, while the blue component undergoes occasional flares. While its visual and photographic light changes considerably, it seems that the bolometric luminosity is nearly constant. A flare of $Z$ Andromedae in 1939 and again in 1961 completely suppressed the late-type absorption features as well as the highly excited emission lines. Instead, a strong blue continuum dominated the spectrum and displayed absorption lines typical for early A stars.

More revealing is the history of AG Pegasi (Boyarchuk, 1967). The star brightened up as a very slow nova between 1855 and 1885, increasing its visual brightness by about 3 mag. The decline has been even slower and today the star is about as bright as it was before the flare. Between 1893 and 1920, its spectrum was that of a Be star with P-Cygni type emissions and numerous absorptions. By 1921, the absorption spectrum weakened a great deal, while the emission lines strengthened. Bright lines showed gradually increasing degree of excitation: today He II $\lambda 4686$ and $\mathrm{N}$ IV $\lambda$ 
4057 lines are among the dominant features, and also the forbidden [O III] and [Ne III] lines are strong. The B-type absorption spectrum has disappeared. Instead, first traces of a late-type spectrum were noted in 1930, and today many features of an M-type spectrum are easily visible. Thus AG Pegasi has behaved on the whole similarly to the other symbiotic objects, only the flare has been much slower.

What is the cause of this behavior? There is little doubt today that AG Pegasi is a binary star. Mean variation of radial velocites, as well as of various spectral features, indicates a period of about 800 days (Boyarchuk, 1967, 1969; Hutchings and Redman, 1972). The complex character of many lines did not permit in the past to determine the mass ratio, but the flare disturbance has settled down sufficiently to make the prospects much better in the near future.

The spectral changes of AG Pegasi can be interpreted as the formation of a shell around the hot star. The shell was originally of small size and optically thick, but as it expanded it gradually became optically thin. Today it has the form of a nebula of such a low density and large size that the strong ultraviolet radiation from the hot component can penetrate it to a great distance and produce emission lines of high excitation. If this picture is basically correct, what was the cause of the flare? If this flaring up is an intrinsic property of the hot component, then why is it always accompanied, in symbiotic variables, by a red star? It is more natural to assume that the outbursts of the blue star are triggered by material flowing to it from the unstable late-type component. The process of mass ejection may well be intermittent or fluctuating on the time scale given by our observations. Indeed, convection in the envelopes of red giants itself must lead to statistical fluctuations in the rate of mass loss by convective overshoot. However, I think that the most important cause of flaring is that conditions of instability build up gradually in the blue star. I think that symbiotic variables may not be basically too different from novae and $U$ Geminorum stars; the term 'nova-like variables' is probably more meaningful than one might think at first sight.

What is the nature of the components in a symbiotic binary such as AG Pegasi? The cool component probably is typically an $\mathbf{M}$ giant. Hutchings and Redman (1972) found that the late-type spectrum is not quite normal. Perhaps we should not expect a fully normal spectrum in an atmosphere which is losing mass at a high rate, and moreover is illuminated by a hot star. It was very encouraging to see that our calculations of mass loss for the model of AX Monocerotis described in Chapter 5 (Plavec and Polidan, to be published) gave effective temperatures corresponding to an M-type giant at the phases of mass loss - both rapid and slow. At later stages of mass loss, the abundances are no longer normal.

What is the nature of the blue component? It is much hotter than a main sequence star of spectral type B. Boyarchuk $(1966,1967,1969)$ suggests that the blue component of AG Pegasi is a Wolf-Rayet star, WN 6. Perhaps the term 'Wolf-Rayet star' should not be taken too literally in this context. By Boyarchuk's own definition of the symbiotic variables, the emission lines are relatively narrow, less than $100 \mathrm{~km} \mathrm{~s}^{-1}$. This is much less than the typical width of the emission bands in Wolf-Rayet stars which 
corresponds to $500 \mathrm{~km} \mathrm{~s}^{-1}$ or $1000 \mathrm{~km} \mathrm{~s}^{-1}$. Moreover, blue-shifted absorption components are typical for the W-R stars but not for the symbiotic variables.

I suggest that the blue components are helium stars near the helium main sequence. According to Boyarchuk (1969), in Z Andromedae the radius of the blue component varies between about 0.25 and 0.75 solar radii, and the visual absolute magnitude between $+1^{M}$ and $+3^{M}$. These values certainly do not indicate $\mathrm{W}-\mathrm{R}$ character. Rather, they remind us of Paczyński's models of pure helium stars of about $2 M_{\odot}$ (Paczyński, 1971b). The hot component of AG Pegasi may have a similar mass. Since the radial velocity curves indicate a mass ratio of about 5:1 in favor of the red component, this star may be a giant of about $10 M_{\odot}$. With a period of $800 \mathrm{~d}$, the orbital radius would then be $820 R_{\odot}$, and if the red giant fills its critical Roche lobe, its mean radius is $430 R_{\odot}$. A star of $10 M_{\odot}$ reaches this radius near the time of carbon ignition. If we cut the masses of both components to one half, which is probably still acceptable, the orbital radius will be $650 R_{\odot}$ and the radius of the giant about $340 R_{\odot}$. Again, a $5 M_{\odot}$ expands to this size on the second giant branch. Long periods of other symbiotic viables indicate a similar picture. Out of five stars sufficiently well studied, four show pariodic variations of radial velocities. Besides AG Pegasi, they are: BF Cygni (period 750 d), RW Hydrae (370 d), and R Aquarii (740 d) (Boyarchuk, 1969). These periods indicate that at least some sybiotic stars, including AG Pegasi, may be examples of case $\mathrm{C}$ of mass transfer.

It would be, of course, already a second process of mass transfer in the system, because we must postulate a previous one, probably of mode $\mathrm{B}$, to account for the presence of the helium star. As was shown in Chapter 7 when we discussed the heliumrich binaries, lifetimes of the two components must be seriously considered before we can decide whether the model does not contain contradictions already in its crudest form. With the great uncertainty as to the masses, and as to the possible mass loss from the system, such a discussion appears premature. What is badly needed now are better data on at least one system. AG Pegasi appears to be in a suitable phase now, and an effort to derive the mass ratio should certainly be made.

\section{Conclusion}

I have attempted to offer an evolutionary interpretation of several groups of close binary stars. In doing this, I acted contrary to Dr. Huang's wise advice "not to stick one's neck out". I think precisely this must be done. I hope that my remarks will stimulate new research, both observational and theoretical, on the problems discussed here. If this happens, most of the models I have proposed will probably be changed or discarded entirely. Yet it is necessary to distinguish between two possible ways in which this can happen. Either it will be found possible to maintain the fundamental assumptions, and the theory of a large-scale mass transfer and/or mass loss will be further developed. Or it will be found that the principles are wrong as well, and we will have to start right from the beginning again. I don't know which alternative is more exciting. 
I have neglected in my talk such important classes of binary stars like the novae, stars of the U Geminorum type, supergiant systems like VV Cephei, the W Ursae Maioris stars, and of course systems like $\beta$ Lyrae, $\varepsilon$ Aurigae and others, suspected by some of harboring black holes. These classes of binary stars are very popular now, and very good review papers already exist or again none can be written since almost every week there is a new model proposed. The clue to the mystery of these very popular systems may well be found in a patient study of the less conspicuous but simpler ones. The basic configurations will probably not be too many. Therefore, whichever system you pick up, you are contributing to one of the most exciting areas of contemporary astrophysics.

\section{Acknowledgements}

I wish to thank Mr. R. S. Polidan for his help with computations and diagrams, and Miss J. Kuebler for careful typing.

The work on this paper and the original investigations reported in it have been supported by grant GP-32886X from the National Science Foundation.

\section{References}

Bahcall, J. N. and Bahcall, N. A.: 1972, IAU Circ., No. 2427.

Bath, G. T.: 1969, Astrophys. J. 158, 571.

Bath, G. T.: 1972, Astrophys. J. 173, 121.

Batten, A. H.: 1967, Publ. Dominion Astroph. Obs. 13, No. 8.

Benson, R. S.: 1970, Bull. Am. Astron. Soc. 2, 295.

Bessell, M. S.: 1972, Astrophys. J. 175, L133.

Biermann, P. and Kippenhahn, R.: 1971a, Veröffentl. Remeis-Sternw., Bamberg 9, 54.

Biermann, P. and Kippenhahn, R.: 1971b, Astron. Astrophys. 14, 32.

Biermann, P. and Thomas, H. C.: 1971, Veröffentl. Remeis-Sternw., Bamberg 9, 285.

Bolton, C. T.: 1972a, Nature 235, 271.

Bolton, C. T.: 1972b, IAU Circ., No. 2424.

Boyarchuk, A. A.: 1966, Soviet Astron. 10, 783.

Boyarchuk, A. A.: 1967, Soviet Astron. 11, 8.

Boyarchuk, A. A. 1959, Soviet Astron. 3, 748.

Boyarchuk, A. A.: 1969, in L. Detre (ed.), Non-Periodic Phenomena in Variable Stars, D. Reidel Publ. Co., p. 395.

Boyarchuk, A. A. and Pronik, I. I.: 1967, Izv. Krymsk. Astrofiz. Obs. 37, 236.

Brucato, R. J. and Kristian, J.: 1972, IAU Circ., No. 2421.

Cester, B. and Pucillo, M.: 1972, preprint.

Cowley, A. P.: 1964, Astrophys. J. 139, 817.

Cowley, A. P.: 1967, Astrophys. J. 147, 609.

Cowley, A. P. and Gugula, E.: 1972, preprint.

Danziger, I. J., Wallerstein, G., and Böhm-Vitense, E.: 1967, Astrophys. J. 150, 239.

Delplace, A. M.: 1970, Astron. Astrophys. 7, 459.

Eggen, O. J., Kron, G. E., and Greenstein; J. L.: 1951, Publ. Astron. Soc. Pacific 62, 171.

Faulkner, J.: 1971, Astrophys. J. 170, L99.

Gaposchkin, S.: 1945, Astron. J. 51, 109.

Hack, M.: 1967, in M. Hack (ed.), Modern Astrophysics, 163.

Hack, M. and Job, F.: 1965, Z. Astrophys. 62, 203.

Harmanec, P.: 1970a, Bull. Astron. Inst. Czech. 21, 113.

Harmanec, P.: 1970b, Bull. Astron. Inst. Czech. 21, 316. 
Harmanec, P., Koubský, P., and Krpata, J.: 1972, Astrophys. Letters 11, 119.

Hazlehurst, J. and Meyer-Hoffmeister, E.: 1971, Veröffentl. Remeis-Sternw., Bamberg 9, 289.

Hickock, J.: 1971, preprint.

Horn, J.: 1971, Bull. Astron. Inst. Czech. 22, 37.

Horn, J., Kříž, S., and Plavec, M.: 1969, Bull. Astron. Inst. Czech. 20, 193.

Horn, J., Křǐž, S., and Plavec, M.: 1970, Bull. Astron. Inst. Czech. 21, 45.

Hubbard, W. B., and Wagner, R. L.: 1970, Astrophys. J. 159, 93.

Hutchings, J. B. and Redman, R. O.: 1972, Publ. Astron. Soc. Pacific 84, 240.

Hynek, A. J.: 1940, Contr. Perkins Obs., No. 14.

Iben, I.: 1967, Ann. Rev. Astron. Astrophys. 5, 571.

Kippenhahn, R. and Weigert, A.: 1967, Z. Astrophys. 65, 251.

Kippenhahn, R., Kohl, K., and Weigert, A.: 1967, Z. Astrophys. 66, 58.

Kopal, Z.: 1955, Ann. Astrophys. 18, 379.

Kopal, Z.: 1971, Publ. Astron. Soc. Pacific 83, 521.

Kopal, Z. and Shapley, M. B.: 1956, Jodrell Bank Ann. 1, 141.

Kraft, R. P.: 1958, Astrophys. J. 127, 625.

Kraft, R. P.: 1972, Contr. Lick Obs., No. 368.

Kříž, S.: 1969a, Bull. Astron. Inst. Czech. 20, 127.

Křiž, S.: 1969b, in M. Hack (ed.), Mass Loss from Stars, D. Reidel Publ. Co., p. 257.

Kruszewski, A.: 1966, Adv. Astron. Astrophys. 4, 233.

Lamb, D. Q. and Sorvari, J. M.: 1972, IAU Circ. No. 2422.

Lauterborn, D.: 1970, Astron. Astrophys. 7, 150.

Lauterborn, D. and Weigert, A.: 1972, Astron. Astrophys. 18, 294.

Liller, W.: 1972, IAU Circ., No. 2415.

McCluskey, G. E. and Kondo, Y.: 1972, preprint.

Moss, D. L.: 1971, Monthly Notices Roy. Astron. Soc. 153, 41.

Nariai, K.: 1963, Publ. Astron. Soc. Japan 15, 7.

Nariai, K.: 1967, Publ. Astron. Soc. Japan 19, 564.

Paczyński, B.: 1965, Acta Astron. 15, 89.

Paczyński, B.: 1956b, Acta Astron. 15, 197.

Paczyński, B.: 1970, Acta Astron. $20,47$.

Paczyński, B.: 1971a, Ann. Rev. Astron. Astrophys. 9, 183.

Paczyński, B.: 1971b, Acta Astron. $21,1$.

Paczyński, B. and Ziółkowski, J.: 1967, Acta Astron. 17, 7.

Paczyński, B., Ziółkowski, J., and Żytkow, A.: 1969, in M. Hack (ed.), Mass Loss from Stars, D. Reidel Publ. Co., p. 237.

Paczyński, B. and Sienkiewicz, R.: 1972, preprint.

Peters, G. J.: 1972, Publ. Astron. Soc. Pacific 84, 334.

Péton, A.: 1971, Compt. Rend. Acad. Sci. Paris, B 273, 1062.

Prendergast, K. H. and Burbidge, G. J.: 1968, Astrophys. J. 151, L83.

Plavec, M.: 1964, Bull. Astron. Inst. Czech. 15, 156.

Plavec, M.: 1967, Bull. Astron. Inst. Czech. 18, 334.

Plavec, M.: 1968, Adv. Astron. Astrophys. 6, 201.

Plavec, M.: 1970a, Publ. Astron. Soc. Pacific 82, 957.

Plavec, M.: 1970b, in A. Slettebak (ed.), Stellar Rotation, D. Reidel Publ. Co., p. 133.

Plavec, M. and Horn, J.: 1969, in M. Hack (ed.), Mass Loss from Stars, D. Reidel Publ. Co., p. 242.

Plavec, M., Křǐž, S., and Horn, J.: 1969, Bull. Astron. Inst. Czech. 20, 41.

Popper, D.M. : 1970, in K. Gyldenkerne and R. West (eds.), Mass Loss and Evolution in Close Binaries, p. 20.

Refsdal, S. and Weigert, A.: 1969, Astron. Astrophys. 1, 167.

Sahade, J. (ed.): 1962, Symposium on Stellar Evolution, La Plata, p. 225.

Sahade, J.: 1971, Veröffentl. Remeis-Sternw. Bamberg 9, 285.

Sato, K.: 1971, Publ. Astron. Soc. Japan 23, 335.

Schmidt, H.: 1959, Z. Astrophys. 48, 249.

Schreier, E., Levinson, R., Gursky, H., Kellogg, E., Tananbaum, H., and Giacconi, R.: 1972, Astrophys. J. 172, L79.

Seydel, F. L.: 1929, Publ. Am. Astron. Soc. 6, 278. 
Struve, O.: 1946, Astrophys. J. 104, 253.

Struve, O. and Huang, S.-S.: 1958, in S. Flügge (ed.), Handbuch der Physik, Springer Verlag, 50, p. 269.

Svechnikov, M. A.: 1955, Peremennyje Zvezdy 10, 252.

Tananbaum, H., Gursky, H., Kellogg, E. M., Levinson, R., Schreier, E., and Giacconi, R.: 1972, Astrophys. J. 174, L143.

Underhill, A. B.: 1952, Publ. Dominion Astrophys. Obs. 9, 129.

Underhill, A. B.: 1966, Early-Type Stars, D. Reidel Publ. Co., p. 233.

Van den Heuvel, E. P. J.: 1970, in A. Slettebak (ed.), Stellar Rotation, D. Reidel Publ. Co., p. 178.

Van den Heuvel, E. P. J. and Heise, J.: 1972, Nature Phys. Sci. 239, 67.

Walker, M. F.: 1953, Astrophys. J. $118,481$.

Wallerstein, G., Greene, T. F., and Tomley, L. J.: 1967, Astrophys. J. 245, 150.

Webster, B. L. and Murdin, P.: 1972, Nature 235, 37.

Weigert, A.: 1968, in L. Perek (ed.), Highlights of Astronomy, D. Reidel Publ. Co. p. 414.

Widing, K. G.: 1966, Astrophys. J. 143, 121.

Wilson, R. E.: 1972, preprint.

Wright, K. O.: 1957, Publ. Astron. Soc. Pacific 69, 552.

Ziółkowski, J.: 1969, Astrophys. Space Sci. 3, 14.

Ziółkowski, J.: 1970, Acta Astron. $20,213$. 